J. H. BRAMBLE

A. H. SCHATZ

\title{
Estimates for spline projections
}

Revue française d'automatique, informatique, recherche opérationnelle. Analyse numérique, tome 10, $\mathrm{n}^{\mathrm{O}} 2$ (1976), p. 5-37.

<http://www.numdam.org/item?id=M2AN_1976_10_2_5_0>

(C) AFCET, 1976, tous droits réservés.

L'accès aux archives de la revue "Revue française d'automatique, informatique, recherche opérationnelle. Analyse numérique » implique l'accord avec les conditions générales d'utilisation (http://www.numdam.org/legal. php). Toute utilisation commerciale ou impression systématique est constitutive d'une infraction pénale. Toute copie ou impression de ce fichier doit contenir la présente mention de copyright.

\section{Numdam}

Article numérisé dans le cadre du programme

Numérisation de documents anciens mathématiques

http://www.numdam.org/ 


\title{
ESTIMATES FOR SPLINE PROJECTIONS (1)
}

\author{
by J. H. Bramble $\left({ }^{2}\right.$ ) and A. H. Schatz $\left({ }^{2}\right)$
}

\section{INTRODUCTION}

Let $\Omega$ be a domain in $R^{N}$ and let $S_{r}^{h}(\Omega)$ denote the restrictions to $\Omega$ of tensor products of splines of order $r$ defined on a uniform mesh of size $h$ in $R^{N}$; i. e. in each variable these are piecewise polynomials of order $r-1$ which have $r-2$ continuous derivatives on $R^{N}$. It is well known that if $u$ has $r$ continuous derivatives on $\bar{\Omega}$ then there exists a spline in $S_{r}^{h}(\Omega)$ which approximates $u$ in maximum norm to order $h^{r}$. In this paper we shall investigate various types of interior maximum-norm estimates for a variety of projection methods which use spline subspaces. In particular we shall focus our attention on $L_{2}$ projections and a class of Ritz-Galerkin methods used in approximating solutions of elliptic boundary value problems.

This paper may be thought of as being divided into two parts. Let $\Omega_{0} \subset \subset \Omega_{1} \subset \subset \Omega \subset R^{N}$. In the first part we shall first show that if $u_{h} \in S_{r}^{h}(\Omega)$ is the best $L_{2}$ approximation to $u$ on $\Omega$ and if $u$ has $r$ continuous derivatives on $\Omega_{1}$, then in maximum-norm $u_{h}$ approximates $u$ to order $h^{r}$ on any compact subdomain $\Omega_{0}$ of $\Omega_{1}$. We then consider approximations $u_{h} \in S_{r}^{h}\left(\Omega_{1}\right)$ satisfying a set of interior equations associated with Ritz-Galerkin methods for elliptic boundary value problems. It is then shown that if $r \geqq 3$, i. e. forsplines which are at least piecewise quadratic polynomials, the error in maximum-norm is bounded by a term of order $h^{r}$ on $\Omega_{0}$ (provided $u$ has $r$ continuous derivatives on $\bar{\Omega}_{1}$ ) plus the error in an arbitrary negative norm on $\Omega_{1}$. This last term (which is not present in the case of $L_{2}$ projections) measures the effect on the error on $\Omega_{0}$ due to factors outside of $\Omega_{1}$ (for example the smoothness of $u$ outside of $\Omega_{1}$, the smoothness of the boundary and the way a particular method treats the boundary conditions). Assuming for the moment that this term is of order $h^{r}$ (this is often the case) then our main point here is that locally the error is of order $h^{r}$ (in maximum-norm) if $u$ has $r$ continuous derivatives. Interior $L_{2}$ estimates for best $L_{2}$ approximation were derived

( ${ }^{1}$ This work was supported in part by a grant from the National Science Foundation.

( ${ }^{2}$ ) Department of Mathematics, Cornell Unıversity, Ithaca, N. Y. U.S.A.

Revue Française d'Automatique, Informatique et Recherche Opérationnelle, août 1976. 
in [12] and for Ritz-Galerkin methods in [13]. In [5] maximum-norm interior estimates were derived for Ritz-Galerkin methods, however the local requirements on the smoothness of $u$ are greater.

In the second part of this paper we shall prove some "super-convergence" results. Briefly, let $u_{h}$ be of the form

$$
u_{h}=\sum_{\alpha} C_{\alpha} \psi\left(h^{-1} x-\alpha\right), \quad x \in \Omega,
$$

where $\alpha$ is a multi-integer, and the $\psi\left(h^{-1} x-\alpha\right)$ form a basis for $S_{r}^{h}$. We shall first show that given any non-negative integer $t$ there exist constants $d_{\eta}$ (depending on $t$ ), such that if $u_{h}$ is the $L_{2}$ projection of $u$ onto $S_{r}^{h}$, then at any mesh point $h \gamma$ (dist $(h \gamma, \partial \Omega) \geqq C h), \sum_{\alpha} C_{\alpha} d_{\gamma-\alpha}$ approximates $u(h \gamma)$ of order $h^{2 t}$ provided $u$ is sufficiently smooth. The constants $d_{\eta}$ have the following properties:

(i) They are easily computable and depend only on $\psi$ and $t$.

(ii) All but a finite number of the $d_{\eta}$ vanish.

Suppose now that we choose the $d_{\eta}$ corresponding to $t=2 r-2$ and $u_{h}$ satisfies the aforementioned interior Ritz-Galerkin equations corresponding to an elliptic boundary value problem on $\Omega_{1}$. We shall then show that if $r \geqq 3$ and $h \gamma \in \Omega_{0}$, then $\sum C_{\alpha} d_{\gamma-\alpha}$ approximates $u(h \gamma)$ of order $h^{2 r-2}(\ln 1 / h)$ plus a term bounded by the error in any negative norm on $\Omega_{1}$. In many applications this last term is of order $h^{2 r-2}$. Hence in those cases, a simple linear combination of the $C_{\alpha}$ 's gives order $h^{2 r-2}(\ln 1 / h)$ at mesh points. It will then be shown that if $r \geqq 3$ and odd, then for $h \gamma \in \Omega_{0}, u_{h}(h \gamma)$ approximates $u(h \gamma)$ to order $h^{r+1}(\ln 1 / h)$ plus the error in an arbitrary negative norm on $\Omega_{1}$.

In proving some of the above superconvergence results we make use of the following which we feel is of independent interest: Let $P_{0} u$ and $P_{1} u$ denote the $L_{2}$ and $H^{1}$ (energy norm) projection of $u$ onto $S_{r}^{h}\left(R^{N}\right)$, where $u \in C^{2 r-2}$ on $R^{N}$ and has compact support. Then in maximum-norm on $R^{N}, P_{0} u$ approximates $P_{1} u$ of order $h^{2 r-2}(\ln 1 / h)$ for $r \geqq 3$.

An outline of the paper is as follows: In Section 2 we introduce notation and preliminaries. In Section 3 we prove a maximum-norm error estimate for the best $L_{2}$ approximation on $R^{N}$. This result was first proved by Fix and Strang [10]. Our method of proof relies on Fourier multipliers and differs from theirs. In Section 4 a sharp maximum-norm error estimate for $H^{1}$ (energy norm) projections on $R^{N}$ is obtained. In Section 5 and 6 we use the results of Sections 3 and 4 to obtain interior maximum-norm error estimates for best $L_{2}$ approximations and for a class of Ritz-Galerkin methods mentioned above. In Section 7 we obtain a superconvergence result in maximumnorm for the comparison of $L_{2}$ and $H^{1}$ (energy norm) projections on $R^{N}$. 
In Section 8 superconvergence results at interior mesh points for $L_{2}$ projections and a class of Ritz-Galerkin equations by "averaging" the computed coefficients are considered. In Section 9 we investigate the question of superconvergence at interior mesh points for $L_{2}$ projections and a class of Ritz-Galerkin equation without averaging. In Sections $10,11,12$, and 13 we exemplify the theory in special cases. In one of our examples we consider the interior rate of convergence in maximum-norm of the approximate Green's function to the Green's function. We show that away from the singular point the rate of convergence is of order $h^{r}$. We wish to emphasize that the technique used in the proof is not special to the spline functions discussed in this paper nor the particular Ritz-Galerkin method used. It relies only on the fact that good interior maximum-norm and certain negative-norm estimates are available, the former being proved here for special spline subspaces. We then apply this result to derive order $h^{r}$ estimates in regions where $u$ is smooth but outside of which it might be quite badly behaved. This seems to indicate that the effects on the interior rates of convergence for Ritz-Galerkin methods due to either a nonsmooth boundary or a non-smooth right hand side are quite different.

\section{PRELIMINARIES}

Let $\Omega$ be an open subset of $R^{N}, N$-dimensional Euclidean space. Let $C^{k}(\Omega)$ be the set of complex valued functions on $\Omega$ which have continuous partial derivatives of order at least $k$, which are bounded on $\Omega$. On $C^{k}(\Omega)$ we introduce the norm

$$
|v|_{k, \Omega}=\sup _{\substack{x \in \Omega \\|\alpha| \leqq k}}\left|D^{\alpha} v(x)\right|
$$

where $\alpha$ is a multi-index and $D^{\alpha}=\partial_{\alpha_{1}} / \partial x_{1}^{\alpha_{1}} \ldots \partial_{\alpha_{N}} / \partial x_{N}^{\alpha_{N}}$ and $|\alpha|=\sum_{j=1}^{N} \alpha_{j}$. If $\Omega$ is bounded then by $H^{k}(\Omega)$ for $k$ a non-negative integer we shall mean the usual Sobolev space of order $k$; i. e. $H^{k}(\Omega)$ is the closure of $C^{k}(\Omega)$ in the norm

$$
\|v\|_{k, \Omega}=\left(\sum_{|\alpha| \leqq k} \int_{\Omega}\left|D^{\alpha} v\right|^{2} d x\right)^{1 / 2} .
$$

If $\Omega=R^{N}$, then $H^{k}(\Omega)$ will be the complection of $C_{0}^{k}(\Omega)$ under the norm (2.1). Here $C_{0}^{k}(\Omega)$ consists of those functions in $C^{k}(\Omega)$ with support contained in $\Omega$ and compact. We shall denote the norms on $C^{k}\left(R^{N}\right)$ and $H^{k}\left(R^{N}\right)$ by $|\cdot|_{k}$ and $\|\cdot\|_{k}$. Note that $H^{0}(\Omega)=L_{2}(\Omega)$.

We shall also need the following norms: For $v \in L_{2}(\Omega)$ :

$$
\|v\|_{-k, \Omega}=\sup _{\varphi \in C_{0}^{k}(\Omega)} \frac{\left|(v, \varphi)_{\Omega}\right|}{\|\varphi\|_{k, \Omega}}
$$

août 1976. 
where $(v, \varphi)_{\Omega}$ is the $L_{2}$-inner product. We also set

$$
\|\| v \|_{-k, \Omega}=\sup _{\varphi \in C^{\prime}(\Omega)} \frac{\left|(v, \varphi)_{\Omega}\right|}{\|\varphi\|_{k, \Omega}} .
$$

REMARK: The above definitions of norms may be extended to all real values of the indices by appealing to the theory of interpolation spaces (cf. [7]). For the sake of simplicity we shall not discuss this generalization and thus to be precise will consider throughout this paper only integer values for the norm indices.

For a given positive integer $r$ we denote by $\psi(x)$ the $B$-spline of order $r$ (cf. [14]). This function is the $r-1$ fold convolution of the characteristic function of the unit cube with itself. More precisely let

$$
\chi(t)=\left\{\begin{array}{l}
1,|t| \leqq 1 / 2 \\
0,|t|>1 / 2
\end{array}\right.
$$

and $\chi^{(r)}=(\chi * \ldots * \chi)$, where $*$ denotes convolution. Then

$$
\widetilde{r-1 \text { times }}(x)=\prod_{j=1}^{N} \chi^{(r)}\left(x_{j}\right) .
$$

Since $\psi$ will be so frequently used and $r$ will always be fixed we have suppressed the dependence on $r$ in the notation.

Denote by $Z^{N}$ the multi-integers and let $h$ be a positive number less than $I$.

Definition: For $r$ a positive integer and $h$ given with $0<h<1$, a function $v$ of the form

$$
v(x)=\sum_{\alpha \in Z^{N}} v_{\alpha} \psi\left(h^{-1} x-\alpha\right)
$$

is called a spline of order $r$. The totality of all such functions will be denoted by $S^{h}$.

Note that since the support of $\psi$ is compact, for any $x$ the sum in (2.2) contains only a finite number of non-vanishing terms so that no restriction need be placed on the $v_{\alpha}$. The splines of order $r$ have the following well known properties:

(a) They are piecewise polynomials of degree $r-1$.

(b) They are of class $C^{r-2}$.

In addition to these, the splines possess some important approximation theoretic properties. We summarize these as follows. 
Lemma 2.1: Let $v \in H^{s}(\Omega)$ with $0 \leqq s \leqq r$. Then there exists a constant $C$ and $v_{h} \in S^{h}$ such that

$$
\left\|v-v_{h}\right\|_{j, \Omega} \leqq C h^{s-j}\|v\|_{s, \Omega}
$$

for $0 \leqq j \leqq s, j \leqq r-1$. The constant $C$ does not depend on $v$.

For the proof of this, $c f$. [9] or [2].

In addition to this the splines also have similar properties relative to the norms on $C^{k}$.

Lemma 2.2: Let $v \in C^{s}$ with $0 \leqq s \leqq r$. Then there exists a constant $C$, independent of $v$, and $Q_{h} v$ in $S^{h}$ such that

$$
\left|v-Q_{h} v\right|_{0} \leqq C h^{s}|v|_{s}
$$

Further, if $v \in C_{0}^{s}$ then $Q_{h} v \in C_{0}^{0}$.

For a proof of this, $c f$. [6].

Let $f \in L_{p}$ with $1 \leqq p \leqq 2$. Then the Fourier transform of $f$ is given by

$$
\hat{f}(\xi)=\int_{R^{N}} f(x) e^{-i\langle x, \xi\rangle} d x, \text { a. e. }
$$

where $\langle x, \xi\rangle=\sum_{j=1}^{N} x_{j} \xi_{j}(c f .[15])$. The inverse transform $\breve{f}(\xi)$ is given by

$$
\check{f}(\xi)=\frac{1}{(2 \pi)^{N}} \hat{f}(-\xi)
$$

and if $\hat{f} \in L_{p}, 1 \leqq p \leqq 2$ then $\breve{\hat{f}}=f$. It is also well known (cf. [16]) that there exists a constant $C$ such that for $1<, p \leqq 2,(1 / p)+\left(1 / p^{\prime}\right)=1$ and $f \in L_{p}$,

$$
\left(\int_{R^{N}}|\hat{f}|^{p^{\prime}} d x\right)^{1 / p^{\prime}} \leqq C\left(\int_{R^{N}}|f|^{p} d x\right)^{1 / p}
$$

Let $m$ be a bounded measurable function and $f \in L_{2}$. Then the transformation

$$
T_{m} f=(\hat{f} m)^{2}
$$

is called a Fourier multipier transformation (cf. [15]). Notice that $T_{m} f \in L_{2}$

Definition: A multiplier $m$ belongs to the class $\mathscr{M}_{p}$ for $1 \leqq p \leqq \infty$ if there exists a constant $M_{p}$ such that for $f \in L_{2} \cap L_{p}$ :

$$
\left(\int_{R^{N}}\left|T_{m} f\right|^{p} d x\right)^{1 / p} \leqq M_{p}\left(\int_{R^{N}}|f|^{p} d x\right)^{1 / p} \quad \text { if } \quad 1 \leqq p<\infty
$$

août 1976. 
or

$$
\left|T_{m} f\right|_{0} \leqq M_{\infty}|f|_{0} \quad \text { if } \quad p=\infty .
$$

It is well known that for $(1 / p)+\left(1 / p^{\prime}\right)=1, \mathscr{M}_{p}=\mathscr{M}_{p^{\prime}}$. We shall be mainly concerned here with multipliers in $\mathscr{M}_{\infty}$. The following gives a simple condition under which $m \in \mathscr{M}_{\infty}$.

Lemma 2.3: Suppose that $\left[\prod_{j=1}^{N}\left(1+\partial / \partial \eta_{j}\right) m\right] \in L_{p}$ for some $p$ with $1<p \leqq 2$. Then $m \in \mathscr{M}_{\infty}$.

Proof: For $f \in L_{2} \cap C^{0}$

$$
T_{m} f(x)=(f * \hat{m})(x) .
$$

Hence it is clearly sufficient to show that $\hat{m} \in L_{1}$. Now

$$
\int_{R^{N}}|\hat{m}(x)| d x=\int_{R^{N}}\left|\prod_{j=1}^{N}\left(1-i x_{j}\right)\right|^{-1}\left|\left(\prod_{j=1}^{N}\left(1+\frac{\partial}{\partial \eta_{j}}\right) m\right)^{n}\right| d x .
$$

Let $1<p \leqq 2$. Then by Hölder's inequality

$\int_{R^{N}}|\hat{m}(x)| d x \leqq\left(\int_{R^{N}} \frac{d x}{\prod_{j=1}^{N}\left(1+x_{j}^{2}\right)^{p / 2}}\right)^{1 / p}\left(\int_{R^{N}}\left|\left(\prod_{j=1}^{N}\left(1+\frac{\partial}{\partial \eta_{j}}\right) m\right)^{\wedge}\right|^{p^{\prime}} d x\right)^{1 / p^{\prime}}$.

The lemma now follows from (2.3).

\section{THE $L_{2}$-PROJFCTION ON $R^{N}$}

Let $u \in C_{0}^{r}$. We define $P_{0} u \in S^{h}$ as the $L_{2}$ projection of $u$ onto $S^{h} \cap L_{2}$; i. e.,

$$
\left(u-P_{0} u, \varphi\right)=0, \quad \forall \varphi \in S^{h} \cap L_{2} .
$$

Note that although $P_{0}$ depends on $h$ and $r$ we have suppressed this in the notation.

Now since $P_{0} u \in S^{h}$, it is given by

$$
P_{0} u(x)=\sum_{\alpha \in Z^{N}} u_{\alpha}^{0} \psi\left(h^{-1} x-\alpha\right) .
$$

We now prove the following.

Lemma 3.1: Let $u \in C_{0}^{r}$ and set $u_{h}(x)=u(h x)$. Then

$$
u_{\alpha}^{0}=\frac{1}{(2 \pi)^{N}} \int_{R^{N}} \hat{u}_{h}(\eta) m_{0}(\eta) e^{i\langle\alpha, \eta\rangle} d \eta,
$$


where

and $m_{0} \in \mathscr{M}_{\infty}$.

$$
m_{0}(\eta)=\hat{\psi}(\eta) / a_{0}(\eta), \quad a_{0}(\eta)=\sum_{\beta \in Z^{N}}|\hat{\psi}(\eta+2 \pi \beta)|^{2}
$$

Proof: It follows from (3.1) that

$$
\sum_{\gamma \in Z^{N}} u_{\gamma}^{0}\left(\psi\left(h^{-1} x-\gamma\right), \psi\left(h^{-1} x-\beta\right)\right)=\left(u, \psi\left(h^{-1} x-\beta\right)\right)
$$

and by changing variables

$$
\sum_{\gamma \in Z^{N}} u_{\gamma}^{0}(\psi * \psi)(\gamma-\beta)=\left(u_{h} * \psi\right)(\beta)
$$

Multiplying by $e^{-i\langle\beta, \eta\rangle}$ and summing over $\beta$ we obtain

$$
\left(\sum_{\gamma \in Z^{N}} u_{\gamma}^{0} e^{-i\langle\gamma, \eta\rangle}\right)\left(\sum_{\beta \in Z^{N}}(\dot{\psi} * \psi)(\beta) e^{-i\langle\beta, \eta\rangle}\right)=\sum_{\beta \in Z^{N}}\left(u_{h} * \psi\right)(\beta) e^{-i\langle\beta, \eta\rangle} .
$$

Applying the Poisson summation formula we obtain

$$
\left(\sum_{\gamma \in Z^{N}} u_{\gamma}^{0} e^{-i\langle\gamma, \eta\rangle}\right) a_{0}(\eta)=\sum_{\beta \in Z^{N}} \hat{u}_{h}(\eta+2 \pi \beta) \hat{\psi}(\eta+2 \pi \beta)
$$

Now

$$
u_{\alpha}^{0}=\frac{1}{(2 \pi)^{N}} \int_{C_{\pi}}\left(\sum_{\gamma \in Z^{N}} u_{\gamma}^{0} e^{-i\langle\gamma, \eta\rangle}\right) e^{i\langle\alpha, \eta\rangle} d \eta,
$$

where $C_{\pi}=\left\{\eta|| \eta_{j} \mid \leqq \pi, j=1, \ldots, N\right\}$.

Hence from (3.3) and (3.4) we have

$$
u_{\alpha}^{0}=\frac{1}{(2 \pi)^{N}} \int_{|\eta,| \leq \pi} \frac{\sum_{\beta \in Z^{N}} \hat{u}_{h}(\eta+2 \pi \beta) \hat{\psi}(\eta+2 \pi \beta)}{a_{0}(\eta)} e^{i\langle\alpha, \eta\rangle} d \eta .
$$

Using the periodicity of $a_{0}(\eta)$ and the exponential, (3.2) follows by changing variables.

That $m_{0} \in \mathscr{M}_{\infty}$ follows from the form of $m_{0}$. Now $m_{0}=\hat{\psi} / a_{0}$, where

Hence

$$
\hat{\psi}(\eta)=\prod_{j=1}^{N}\left(\frac{\sin \eta_{j} / 2}{\eta_{j} / 2}\right)^{r}
$$

$$
m_{0}(\eta)=\prod_{j=1}^{N}\left[\left(\frac{\sin \eta_{j} / 2}{\eta_{j} / 2}\right)^{r} / \sum_{l \in Z^{1}}\left(\frac{2 \sin \eta_{j} / 2}{\eta_{j}+2 \pi l}\right)^{2 r}\right] .
$$

Since $\sum_{l \in Z^{1}}(2 \sin t / 2) /(t+2 \pi l)^{2 r}$, for $t \in R^{1}$, is a smooth periodic function which never vanishes, in order to satisfy the conditions of Lemma 2.3 , it is août 1976. 
enough to observe that $((\sin t / 2) / t / 2)^{r}$ and its first derivative are in $L_{2}\left(R^{1}\right)$. Hence $m_{0} \in \mathscr{M}_{\infty}$.

The previous theorem provides us with the basis for a different proof of a result first proved by Fix and Strang [10].

THEOREM 1: Let $u \in C_{0}^{s}$ with $0 \leqq s \leqq r$. Then there is a constant $C$ independent of $u$ such that for any $h, 0<h<1$,

$$
\left|u-P_{0} u\right|_{0} \leqq C h^{s}|u|_{s}
$$

Proof: Let $Q_{h} u \in S^{h}$ be given by Lemma 2.2. Then

$$
\left|u-P_{0} u\right|_{0} \leqq|E|_{0}+\left|P_{0} E\right|_{0},
$$

where $E=Q_{h} u-u$. Now

and hence

$$
\left(P_{0} E\right)(x)=\sum_{\alpha \in Z^{N}} E_{\alpha}^{0} \psi\left(h^{-1} x-\alpha\right)
$$

$$
\left|P_{0} E\right|_{0} \leqq \sup _{\alpha \in Z^{N}}\left|E_{\alpha}^{0}\right|
$$

By Lemma 3.1:

$$
E_{\alpha}^{0}=\frac{1}{(2 \pi)^{N}} \int_{R^{N}} \hat{E}_{h} m_{0} e^{i\langle\alpha, \eta\rangle} d \eta,
$$

and since $m_{0} \in \mathscr{M}_{\infty}$,

$$
\left|E_{\alpha}^{0}\right| \leqq C|E|_{0}
$$

From (3.5), (3.6), and (3.7) it follows that

$$
\left|u-P_{0} u\right|_{0} \leqq C|E|_{0}
$$

The theorem follows now from Lemma 2.1.

\section{4. $H^{1}$-PROJECTIONS ON $R^{N}$}

Let $D(v, w)=\sum_{j=1}^{N}\left(\partial v / \partial x_{j}, \partial w / \partial x_{j}\right)$. We want to consider the projection operator $P_{1} u \in S^{h} \cap H^{1}$ for $u \in C_{0}^{s}, s \geqq 1$, where $P_{1}$ is defined by

$$
D\left(u-P_{1} u, \varphi\right)=0, \quad \forall \varphi \in S^{h} \cap H^{1} .
$$

We now prove the following.

Lemma 4.1: Let $r \geqq 3$ and $u \in C_{0}^{s}$ with $s \geqq 1$. Then $P_{1} u \in S^{h} \cap H^{1}$ exists and is unique. It is given by

$$
\left(P_{1} u\right)(x)=\sum_{\alpha \in Z^{N}} u_{\alpha}^{1} \psi\left(h^{-1} x-\alpha\right)
$$


with

where

$$
u_{\alpha}^{1}=\frac{1}{(2 \pi)^{N}} \int_{R^{N}} \hat{u}_{h} m_{1} e^{i\langle\alpha, \eta\rangle} d \eta
$$

$$
m_{1}=\hat{\psi}(\eta)|\eta|^{2} i a_{1}(\eta) \quad \text { and } \quad a_{1}(\eta)=\sum_{x \in Z^{N}}|\psi(\eta+2 \pi \alpha)|^{2}|\eta+2 \pi \alpha|^{2} .
$$

Finally $m_{1} \in \mathscr{M}_{\infty}$.

Proof: By a calculation similar to that made in the proof of Lemma 3.1 the coefficients defined by (4.2) uniquely define a solution of (4.1). The only remaining thing to prove is that $m_{1} \in \mathscr{M}_{\infty}$. The proof in this case is more delicate than that of $m_{0}$ since $m_{1}$ is not a product of functions of one variable. Hence the $N$-dimensional case is not just an obvious generalization of the one dimensional case.

To prove that $m_{1} \in \mathscr{M}_{\infty}$ we shall apply Lemma 2.3 . In order to do this we shall show that

$$
\begin{gathered}
A^{k} m_{1}=\frac{\partial^{k} m_{1}}{\partial \eta_{1} \ldots \partial \eta_{k}} \in L_{p} \quad \text { for } \quad 1<p<1+\frac{1}{N-1}, \\
k=0, \ldots, N \quad \text { with } \quad A^{0}=I .
\end{gathered}
$$

Because of the symmetry of $m_{1}$ it will then follow that

$$
\prod_{j=1}^{N}\left(1+\frac{\partial}{\partial \eta_{j}}\right) m_{1} \in L_{p}, \quad 1<p<1+\frac{1}{N-1}
$$

and hence $m_{1} \in \mathscr{M}_{\infty}$.

We write

$$
m_{1}(\eta)=4 \hat{\psi}(\eta) \frac{|\eta / 2|^{2}}{S^{2}} \frac{S^{2}}{a_{1}^{2}},
$$

where $S^{2}(\eta)=\sum_{j=1}^{N} \sin ^{2} \eta_{j} / 2$ and first calculate $A^{h}\left(i \eta /\left.2\right|^{2} / S^{2}\right)$ for $1 \leqq k \leqq N$.

Now

$$
\begin{aligned}
A^{k}\left(\frac{|\eta / 2|^{2}}{S^{2}}\right)= & \frac{(-1)^{k}}{S^{2 k}}(k-1) ! \\
& \times\left[\frac{k|\eta / 2|^{2 r}}{S^{2}} \prod_{j=1}^{k} \sin \eta_{j} / 2 \cos \eta_{j} / 2-\sum_{l=1}^{k} \eta_{l} / 2 \prod_{j \neq l} \sin \eta_{j} / 2 \cos \eta_{j} / 2\right] .
\end{aligned}
$$

Hence by elementary estimates we have for a suitable constant $C$

$$
\left|A^{k}\left(\left.\frac{\mid \eta / 2}{S^{2}}\right|^{2}\right)\right| \leqq C\left(1+S^{2-k}\right) \quad \text { for } \quad\left|\eta_{j}\right| \leqq \pi, j=1, \ldots N
$$

août 1976. 
If $\eta_{l} i>\pi$ for some $l$ then we have

$$
\left|A^{k}\left(\frac{|\eta / 2|^{2}}{S^{2}}\right)\right| \leqq C \frac{|\eta|^{2}}{S^{2+k}}
$$

Now we obtain by a tedious calculation

$$
\left|A^{k} \psi\right| \leqq C S_{k}, \quad 0 \leqq k \leqq N \quad \text { if }\left|\eta_{j}\right| \leqq \pi, \quad j=1, \ldots, \quad N,
$$

and if $\left|\eta_{l}\right|>\pi$ for some $l$

and

$$
|\hat{\psi}| \leqq C S^{r} \prod_{j=1}^{N} \frac{1}{1+\left|\eta_{j}\right|^{r}}
$$

$$
\left|A^{k} \hat{\psi}\right| \leqq C S^{r-1} \prod_{j=1}^{N} \frac{1}{1+\left|\eta_{j}\right|^{r}}, \quad 1 \leqq k \leqq N .
$$

In order to estimate $A^{k}\left(\hat{\psi}\left(\mid \eta / 2^{2} / S^{2}\right)\right)$ we use Leibniz' rule. Because of symmetry we may observe that the above estimates for $A^{k}\left(\mid \eta / 2^{2} / S^{2}\right)$ and $A^{k} \hat{\psi}$ are valid for any purely mixed derivative of order $k$. Hence in the case that $\left|\eta_{j}\right| \leqq \pi$ for $j=1, \ldots, N$, we combine (4.3) and (4.5) to obtain

$$
\left|A^{k}\left(\hat{\psi} \frac{|\eta / 2|^{2}}{S^{2}}\right)\right| \leqq C\left(1+S^{1-k}\right) \prod_{j=1}^{N} \frac{1}{1+|\eta|^{r-2}} .
$$

But the same estimate (4.8) in the case $\mid \eta_{1}{ }^{\prime}>\pi$ for some $l$ follows from (4.4), (4.6) and (4.7).

We need yet to estimate $A^{k}\left(S^{2} / a_{1}\right)$. To do this we first estimate

$$
A^{k}\left(\frac{a_{1}}{S^{2}}\right)=\sum_{\alpha \in Z^{N}} A^{k}\left[\frac{|\hat{\psi}(\eta+2 \pi \alpha)|^{2}|\eta+2 \pi \alpha|^{2}}{S^{2}(\eta+2 \pi \alpha)}\right],
$$

where we have used the fact that $S(\eta)=S(\eta+2 \pi \alpha)$ for $\alpha \in Z^{N}$. Now $\hat{\psi}^{2}$ is just $(\psi * \psi)^{\wedge}$ so that we may apply the estimate (4.8), interpreting $\psi$ as the $B$-spline of order $2 r$, to each term of the sum in (4.9). Hence, since $r \geqq 3$

$$
\begin{aligned}
A^{k}\left(\frac{a_{1}}{S^{2}}\right) & \leqq C\left(1+S^{1-k}\right) \sum_{\alpha}\left[\prod_{j=1}^{N}\left(\frac{1}{1+\left|\eta_{j}+2 \pi \alpha_{j}\right|^{2 r-2}}\right)\right] \\
& \leqq C\left(1+S^{1-k}\right) .
\end{aligned}
$$

Now (4.10) holds for any purely mixed partial derivative of order $k$. Since $a_{1} / S^{2}$ is bounded away from zero, it follows by an easy induction argument, using Leibniz' rule again that for an appropriate constant $C$

$$
\left|A^{k}\left(\frac{S^{2}}{a_{1}}\right)\right| \leqq C\left(1+S^{1-k}\right)
$$


Again (4.8) and (4.11) are valid for any $k^{\text {th }}$ order purely mixed partial derivative and hence by Leibniz' rule again it follows that

$$
\left|A^{k} m_{1}\right| \leqq C\left(1+S^{1-k}\right) \prod_{j=1}^{N} \frac{1}{1+\left|\eta_{j}\right|^{r-2}} .
$$

Finally we see easily from this estimate that since $r \geqq 3$,

$$
\left|\prod_{j=1}^{N}\left(1+\frac{\partial}{\partial \eta_{j}}\right) m_{1}\right| \leqq C S^{1-N} \prod_{j=1}^{N} \frac{1}{1+\left|\eta_{j}\right|} .
$$

Now for $1<p<1+[1 /(\mathrm{N}-1)]$

$$
\begin{aligned}
& \int_{R^{N}}\left|\prod_{j=1}^{N}\left(1+\frac{\partial}{\partial \eta_{j}}\right) m_{1}\right|^{p} d \eta \\
& \quad \leqq C^{p} \int_{R^{N}} S^{p(1-N)} \prod_{j=1}^{N} \frac{1}{\left(1+\left|\eta_{j}\right|\right)^{p}} d \eta \\
& \leqq C^{p} \sum_{\alpha \in Z^{N}} \int_{C_{\pi}} S^{p(1-N)} \prod \frac{1}{\left(1+\left|\eta_{j}+2 \pi \alpha_{j}\right|\right)^{p}} d \eta .
\end{aligned}
$$

For $\eta \in C_{\pi}$ we have, for an appropriate constant $C$,

$$
\frac{1}{1+\left|\eta_{j}+2 \pi \alpha_{j}\right|} \leqq \frac{C}{1+\left|\alpha_{j}\right|}
$$

and

$$
S^{p(1-N)} \leqq C\left\lceil\left.\eta\right|^{p(1-N)} .\right.
$$

Hence from (4.13), (4.14) and (4.15) we obtain

$$
\begin{aligned}
& \int_{R^{N}}\left|\prod_{j=1}^{N}\left(1+\frac{\partial}{\partial \eta_{j}}\right) m_{1}\right|^{p} d \eta \\
& \leqq C \sum_{\alpha \in Z^{N}} \prod_{j=1}^{N} \frac{1}{\left(1+\left|\alpha_{j}\right|\right)^{p}} \int_{C \pi} \frac{d \eta}{|\eta|^{p(N-1)}} .
\end{aligned}
$$

The right hand side is bounded for $1<p<1+[1 /(N-1)]$. Thus we have, from Lemma 2.3, that $m_{1} \in \mathscr{M}_{\infty}$ and the proof of Lemma 4.1 is complete.

We now prove a maximum-norm estimate for $u \rightarrow P_{1} u$.

Theorem 2: Let $u \in C_{0}^{s}$ with $1 \leqq s \leqq r$ and $r \geqq 3$. Then there is a constant $C$, independent of $u$, such that for any $h$, with $0<h<1$,

$$
\left|u-P_{1} u\right|_{0} \leqq C h^{s}|u|_{s}
$$

août 1976. 
Proof: Let $Q_{h} u \in S_{h}$ be given by Lemma 2.2. Then

$$
\left|u-P_{1} u\right|_{0} \leqq|E|_{0}+\left|P_{1} E\right|_{0},
$$

where $E=Q_{h} u-u$. As in the proof of Theorem 2, since by Lemma 4.1 $m_{1} \in \mathscr{M}_{\infty}$, it follows that

$$
\left|P_{1} E\right|_{0} \leqq C|E|_{0}
$$

Hence from (4.17) and (4.18)

$$
\left|u-P_{1} u\right|_{0} \leqq C|E|_{0} .
$$

The inequality (4.16) now follows from Lemma 2.1.

With only technical changes in the arguments we can prove the following. The details will not be given.

THEOREM 3: Let $u \in C_{0}^{s}$ with $1 \leqq s \leqq r$ and $r \geqq 3$. Let $q$ be a non-negative constant and $P_{1} u \in S^{h} \cap H^{1}$ be defined by

$$
D\left(u-P_{1} u, \varphi\right)+q\left(u-P_{1} u, \varphi\right)=0 \text { for all } \varphi \in S^{h} \cap H^{1} .
$$

Then there is a constant $C$, independent of $u$, such that for all $h$, with $0<h<1$,

$$
\left|u-P_{1} u\right|_{0} \leqq C h^{s}|u|_{s} .
$$

\section{5. $L_{2}(\Omega)$-PROJECTIONS AND INTERIOR ESTIMATES}

Let $\Omega_{0}$ be an open subset of $\Omega$ whose closure is compact and contained in $\Omega$. We write this as $\Omega_{0} \subset \subset \Omega$. Let $S^{h}(\Omega)$ denote the splines of order $r$ restricted to $\Omega$. The space $\dot{S}^{h}(\Omega)$ consists of those elements of $S^{h}(\Omega)$ whose supports are contained in $\Omega$. We shall need the following lemma which may be found in $[5]$.

Lemma 5.1: Let $\Omega_{0} \subset \subset \Omega_{1} \subset \subset \Omega$, and suppose that $u_{h} \in S^{h}(\Omega)$ and satisfies $\left(u_{h}, \varphi\right)=0$ for all $\varphi \in \dot{S}_{h}(\Omega)$. For any positive number $\gamma$, fixed but arbitrary, there is a constant $C_{\gamma}$, independent of $u_{h}$, such that for $h$ sufficiently small

$$
\left|u_{h}\right|_{0, \Omega_{0}} \leqq C_{\gamma} h^{\gamma}\left\|u_{h}\right\|_{0, \Omega_{1}} .
$$

With this lemma we may prove the following:

THEOREM 4: Let $u \in L_{2}(\Omega) \cap C^{s}\left(\Omega_{1}\right), 0 \leqq s \leqq r, r \geqq 1$ and let $P_{0, \Omega} u \in S^{h}$ be the $L_{2}(\Omega)$ orthogonal projection onto $S^{h}(\Omega)$; i. e. $\left(u-P_{0, \Omega} u, \varphi\right)=0$ for all $\varphi \in S^{h}(r)$. Let $\Omega_{0} \subset \subset \Omega_{1} \subset \subset \Omega$. Then there is a constant $C$, independent of $u$, such that for sufficiently small $h$

$$
\left|u-P_{0, \Omega} u\right|_{0, \Omega_{0}} \leqq C h^{s}\left(|u|_{s, \Omega_{1}}+\|u\|_{0, \Omega}\right) .
$$


Proof: Let $\Omega_{0} \subset \subset \Omega_{2} \subset \subset \Omega_{1} \subset \subset \Omega$. We multiply $u$ by a suitably chosen function $\omega \in C_{0}^{\infty}\left(\Omega_{1}\right)$ which is equal to 1 on $\Omega_{2}$ and set $\tilde{u}=\omega u$ on $R^{N}$. Evidently then $\tilde{u} \in C_{0}^{s}$ if $u \in C^{s}\left(\Omega_{1}\right)$ and

$$
|\tilde{u}|_{s} \leqq C|u|_{s, \Omega_{1}}
$$

for an appropriately chosen constant $C$ which does not depend on $u$. Now also

Then

$$
\|\tilde{u}\|_{0} \leqq C\|u\|_{0, \Omega}
$$

$$
\left|u-P_{0, \Omega} u\right|_{0, \Omega_{0}} \leqq\left|\tilde{u}-P_{0} \tilde{u}\right|_{0}+\left|P_{0} \tilde{u}-P_{0, \Omega} u\right|_{0, \Omega_{0}} .
$$

Since $\left(P_{0} \tilde{u}-P_{0, \Omega} u, \varphi\right)=0$ for all $\varphi \in \check{S}^{h}\left(\Omega_{2}\right)$ we obtain from Lemma 5.1, with $\gamma=s$ and (5.2), that

$$
\begin{aligned}
\left|P_{0} \tilde{u}-P_{0, \Omega} u\right|_{0, \Omega_{0}} & \leqq C_{s} h^{s}\left\|P_{0} \tilde{u}-P_{0, \Omega} u\right\|_{0, \Omega_{1}} \\
& \leqq C_{s} h^{s}\left(\|u\|_{0}+\|u\|_{0, \Omega}\right) \\
& \leqq C_{s} h^{s}\|u\|_{0, \Omega} .
\end{aligned}
$$

From Theorem 1 and (5.1) we obtain

$$
\left|\tilde{u}-P_{0} \tilde{u}\right|_{0} \leqq C h^{s}|u|_{s, \Omega_{i}} .
$$

The theorem now follows from (5.3), (5.4) and (5.5).

\section{INTERIOR ESTIMATES FOR RITZ-GALERKIN METHODS}

In order to study the maximum-norm of the error in various approximations to boundary value problems we want to give now a local analogue of Theorem 2. We first need a special case of a lemma which may be found in [3].

LEMMA 6.1: Let $p$ and $q$ be arbitrary positive numbers. Then there exists a constant $C_{p}$ such that, for $\Omega_{0} \subset \subset \Omega_{1} \subset \subset \Omega, h$ sufficiently small and any $u_{h} \in S^{h}(\Omega)$ satisfying

$$
D\left(u_{h}, \varphi\right)+q\left(u_{h}, \varphi\right)=0
$$

for all $\varphi \in \dot{S}_{h}(\Omega)$,

$$
\left|u_{h}\right|_{0, \Omega_{0}} \leqq C\left\|u_{h}\right\|_{-p, \Omega_{1}} .
$$

We may now prove:

Theorem 5: Let $u \in C^{s}\left(\Omega_{1}\right), 1 \leqq s \leqq r, r \geqq 3$ and $P_{1, \Omega} u \in S^{h}(\Omega)$ satisfy

$$
D\left(u-P_{1, \Omega} u, \varphi\right)+q\left(u-P_{1, \Omega} u, \varphi\right)=0 \text { for all } \varphi \in \dot{S}^{h}\left(\Omega_{0}\right) \text {, }
$$

août 1976. 
with $\Omega_{0} \subset \subset \Omega_{1} \subset \subset \Omega$ and $q$ a non-negative constant. Let $p$ be a given positive number. Then there is a constant $C$, independent of $u$, such that for $h$ sufficiently small

$$
\left|u-P_{1, \Omega} u\right|_{0, \Omega_{0}} \leqq C\left(h^{s}|u|_{s, \Omega_{1}}+\| u-\left.P_{1, \Omega} u\right|_{-p, \Omega_{1}}\right) .
$$

Proof: Let $\Omega_{0} \subset \subset \Omega_{2} \subset \subset \Omega_{1}$ and let $\tilde{u}$ be the extension of $u$ of $R^{N}$ given in the proof of Theorem 4 with $\tilde{u}=u$ on $\Omega_{2}$. Then

$$
\left|u-P_{1, \Omega} u\right|_{0, \Omega_{0}} \leqq\left|\tilde{u}-P_{1} \tilde{u}\right|_{0}+\left|P_{1} \tilde{u}-P_{1, \Omega} u\right|_{0, \Omega_{0}} .
$$

Since $D\left(P_{1} \tilde{u}-P_{1, \Omega} u, \varphi\right)=0$ for all $\varphi \in S^{h}\left(\Omega_{2}\right)$ we have from Lemma 6.1 that

$$
\begin{aligned}
\left|P_{1} \tilde{u}-P_{1, \Omega} u\right|_{0, \Omega_{0}} & \leqq C\left\|P_{1} \tilde{u}-P_{1, \Omega} u\right\|_{-p, \Omega_{2}} \\
& \leqq C\left(\left|P_{1} u-\tilde{u}\right|_{0}+\left\|\tilde{u}-P_{1, \Omega} u\right\|_{-p, \Omega_{2}} .\right.
\end{aligned}
$$

The theorem now follows from (6.3) and (6.4) using the estimate of Theorem 2 or Theorem 3.

\section{COMPARISON OF $L_{2}$ AND $H^{1}$ PROJECTIONS}

In Sections 3 and 4 , it was shown that $u-P_{0} u_{\mid 0}=O\left(h^{r}\right)$ and $u-P_{1} u_{\mid 0}=O\left(h^{r}\right)$. Here it will be shown that

$$
\left|P_{0} u-P_{1} u\right|_{0}=O\left(h^{2 r-2} \ln \frac{1}{h}\right)
$$

(for sufficiently smooth $u$ ). This superconvergence type result will be used repeatedly in the following sections.

THEOREM 6: Let $r \geqq 3$. Then there exists a constant $C$ independent of $h$ and $u$ such that

(i) If $u \in C^{l}$ :

$$
\left|P_{0} u-P_{1} u\right|_{0} \leqq C h^{l}|u|_{l}, \quad l=h, \ldots, 2 r-3 .
$$

(ii) If $u \in C^{2 r-2}, N=2$ and $r$ odd, or $N=1$ :

$$
\left|P_{0} u-P_{1} u\right|_{0} \leqq C h^{2 r-2}|u|_{2 r-2} .
$$

(iii) If $u \in C^{2 r-2}$ and supp $(u) \subset \Lambda$ a compact set

$$
\left|P_{0} u-P_{1} u\right|_{0} \leqq C\left(\ln \begin{array}{l}
1 \\
h
\end{array}\right) h^{2 r-2}|u|_{2 r-2} \text {. }
$$


Remark: By an entirely different method we can obtain an estimate for $P_{0} u-P_{1} u$ of order $h^{2 r-2}$ without the hypothesis that $u$ have compact support and without the factor $\ln 1 / h$, which improves the order of convergence in (7.3) in the cases not covered by (7.2). The alternative proof requires a somewhat stronger norm on $u$ on the right hand side. The proof of this will be omitted here since one of our purposes here is to obtain the best norm we can on the right hand side relative to the order of convergence.

Proof: By Lemmas 3.1 and 4.1 we have that

$$
\left|P_{0} u-P_{1} u\right|_{0} \leqq \sup _{\alpha \in Z^{N}} \frac{1}{(2 \pi)^{N}}\left|\int_{R^{N}} \hat{u}_{h}\left(m_{0}-m_{1}\right) e^{i\langle\alpha, \eta\rangle} d \eta\right| .
$$

Let $\tilde{w}(y)$ be a function of the single variable $y$ be such that $\tilde{w}(y)=1$ for $|y|<\pi / 2, \tilde{w}(y) \equiv 0$ for $|y| \geqq \pi$ and $\tilde{w} \in C^{\infty}$. Set $w_{j}=\tilde{w}\left(\eta_{j}\right), j=1, \ldots, N$ and $w=\prod_{j=1}^{N} w_{j}$. We shall now estimate the right hand side of (7.4).

$$
\begin{aligned}
\int_{R^{N}} \hat{u}_{h}\left(m_{0}-m_{1}\right) e^{i\langle\alpha, \eta\rangle} d \eta= & \int_{R^{N}} \hat{u}_{h} w\left(m_{0}-m_{1}\right) e^{i\langle\alpha, \eta\rangle} d \eta \\
& +\int_{R^{N}} \hat{u}_{h}\left(1-\prod_{j=1}^{N} w_{j}\right)\left(m_{0}-m_{1}\right) e^{i\langle\alpha, \eta\rangle} d \eta .
\end{aligned}
$$

Since $1-\prod_{j=1}^{N} w_{j}$ vanishes near the origin, it follows that for the second term on the right hand side of (7.5) we have

$$
\begin{aligned}
& \int_{R^{N}} \hat{u}_{h}\left(1-\prod_{j}^{N} w_{j}\right)\left(m_{0}-m_{1}\right) e^{i\langle\alpha, \eta\rangle} d \eta \\
& =h^{l} \int_{R^{N}}\left(\sum_{j=1}^{N}\left(D_{j}^{l} u\right)_{h} \prod_{i<j} w_{i}\left(1-w_{j}\right) \eta_{j}^{-l}\right)\left(m_{0}-m_{1}\right) e^{i\langle\alpha, \eta\rangle} d \eta .
\end{aligned}
$$

It follows from Lemma 2.3 that for each $j, \prod_{j=1} w_{i}\left(1-w_{j}\right) \eta_{j}^{-l} \in \mathscr{M}_{\infty}$. Since $\mathscr{M}_{\infty}$ is a Banach Algebra and $m_{0}-m_{1} \in \mathscr{M}_{\infty}$ we have that $\prod_{i<j} w_{i}\left(1-w_{j}\right) \eta_{j}^{-l}\left(m_{0}-m_{1}\right) \in \mathscr{M}_{\infty}$. Hence for some appropriate constant $C$ we have

$$
\begin{gathered}
\left|\int \hat{u}_{h}\left(1-\prod_{j=1}^{N} w_{j}\right)\left(m_{0}-m_{1}\right) e^{i\langle\alpha, \eta\rangle} d \eta\right| \leqq C h^{l}|u|_{l}, \\
l=0, \ldots, 2 r-2 .
\end{gathered}
$$

août 1976 . 
Let us now estimate the first term on the right hand side of (7.5). To this end we examine carefully the multiplier $w\left(m_{0}-m_{1}\right)$ near the origin. Now

$$
m_{0}-m_{1}=m_{1}\left[\frac{a_{1}-|\eta|^{2} a_{0}}{|\eta|^{2} a_{0}}\right]=m_{1}\left[\frac{a_{1}^{\prime}-|\eta|^{2} a_{0}^{\prime}}{|\eta|^{2} a_{0}}\right],
$$

where $a_{0}$ and $a_{1}$ are defined in Lemmas 3.1 and 4.1, $a_{0}^{\prime}=a_{0}-|\psi|^{2}$ and $a_{1}^{\prime}=a_{1}-|\eta|^{2}|\hat{\psi}|^{2}$. By inspection we see that there are functions $f_{j}(\eta)$ and $g_{j}(\eta)$ in $C^{\infty}$ such that

$$
a_{0}^{\prime} / a_{0}=\sum_{j=1}^{N}\left(\sin \eta_{j} / 2\right)^{2 r} f_{j}(\eta)
$$

and

$$
|\eta|^{-2} a_{1}^{\prime} / a_{0}=|\eta|^{-2} \sum_{j=1}^{N}\left(\sin \eta_{j} / 2\right)^{2 r} g_{j}(\eta)
$$

Hence

$$
\begin{aligned}
w\left(m_{0}-m_{1}\right)= & \sum_{j=1}^{N} w|\eta|^{-2}\left(\sin \eta_{j} / 2\right)^{2 r} g_{j}(\eta) m_{1} \\
& -\sum_{j=1}^{N} w\left(\sin \eta_{j} / 2\right)^{2 r} f_{j}(\eta) m_{1} .
\end{aligned}
$$

Let $\partial_{j, h}$ be the divided difference operator

$$
\partial_{j, h}=h^{-1}\left[v\left(x_{1}, \ldots, x_{j}+h / 2, \ldots, x_{N}\right)-v\left(x_{1}, \ldots, x_{j}, \ldots, x_{N}\right)\right] .
$$

Now for any positive integer $k, i^{k}\left(\sin \eta_{j} / 2\right)^{k} \hat{u}_{h}=h^{k}\left(\widehat{\partial}_{j, h} u\right)_{h}$. Hence we obtain

$$
\begin{aligned}
\int_{R^{N}} & \hat{u}_{h} w\left(m_{0}-m_{1}\right) e^{i\langle\alpha, \eta\rangle} d \eta \\
= & h^{l} \sum_{j=1}^{N}(-i)^{l} \int_{R^{N}} \widehat{\left(\partial_{j, h}^{l} u\right)_{h}} w\left(\sin \eta_{j} / 2\right)^{2 r-l}|\eta|^{-2} g_{j}(n) m_{1} e^{i\langle\alpha, \eta\rangle} d \eta \\
& +(-1)^{r} h^{2 r} \sum_{j=1}^{N} \int_{R^{N}} \widehat{\left(\partial_{j, h}^{2 r} u\right)} w f_{j} m_{1} e^{i\langle\alpha, \eta\rangle} d \eta \\
= & I_{1}+I_{2} .
\end{aligned}
$$

For each $j$ we have that $w f_{j} m_{1} \in \mathscr{M}_{\infty}$ and therefore

$$
\left|I_{2}\right| \leqq C h^{2 r} \sum_{j=1}^{N}\left|\partial_{j}^{2 r} u\right|_{0} \leqq C h^{l}|u|_{l}, \quad l=0, \ldots, 2 r .
$$


Let us now consider for fixed $j$ one term of $I_{1}$. If $0 \leqq l \leqq 2 r-3$ then $2 r-l \geqq 3$ and it follows that

$$
w\left(\sin \eta_{j} / 2\right)^{2 r-l}|\eta|^{-2} g_{j}(\eta) m_{1} \in \mathscr{M}_{\infty}
$$

Hence for some constant $C$ :

$$
\left|I_{1}\right| \leqq C h^{l}|u|_{l}, \quad j=0, \ldots, 2 r-3 .
$$

Combining the estimates (7.4) through (7.12) we obtain the estimate (7.1).

Let us now prove (7.3). To this end set $l=2 r-2$ in (7.10). Hence $I_{1}$ becomes

$$
I_{1}=h^{2 r-2} \sum_{j=1}^{N} \int_{R^{N}} \widehat{\left(\partial_{j, h}^{2 r-2} u\right)_{h}} w\left(\sin \eta_{j} / 2\right)^{2}|\eta|^{-2} g_{j}(\eta) m_{1} e^{i\langle\alpha, \eta\rangle} d \eta .
$$

Let us fix $j$ and look at a typical term. Set $\left(\partial_{j, h}^{2 r-2} u\right)_{h}=v_{h}$ and

$$
\rho(x)=\int_{R^{N}} \Gamma(x-y) v_{h}(y) d y,
$$

where $\Gamma$ is the fundamental solution of the Laplace equation so that

$$
-\Delta \rho=v_{h} \text { in } R^{N}
$$

Clearly then $|\eta| \hat{\rho}^{2}=\hat{v}_{h}$. Hence for an appropriate constant

$$
\begin{aligned}
& \mid \int_{R^{N}}\left(\widehat{\left.\partial_{j, h}^{2 r-2} u\right)_{h}} w\left(\sin \eta_{j} / 2\right)^{2}|\eta|^{2} g_{j}(\eta) m_{1} e^{i\langle\alpha, \eta\rangle} d \eta \mid\right. \\
& \quad \leqq\left|\int_{R^{N}} \hat{\rho} w\left(\sin \eta_{j} / 2\right)^{2} g_{j}(\eta) m_{1} e^{i\langle\alpha, \eta\rangle} d \eta\right| \\
& \quad \leqq\left|\int_{R^{N}} \widehat{\left(\partial_{j, 1}^{2} \rho\right)} w g_{j}(\eta) m_{1} e^{i\langle\alpha, \eta\rangle} d \eta\right| \leqq C\left|\partial_{j, 1}^{2} \rho\right|_{0}
\end{aligned}
$$

where we have used the fact that $w g_{j} m_{1} \in \mathscr{M}_{\infty}$. Finally, we need to estimate $\left|\partial_{j, 1}^{2} \rho\right| 0$. Using the fact that diam ( $\left.\operatorname{supp} v_{h}\right) \leqq C / h$ for some constant $C$ (depending on the support of $u$ ) we obtain by elementary estimates that

$$
\left|\partial_{j, 1}^{2} \rho\right|_{0} \leqq C \ln 1 / h|v|_{0} \leqq C \ln 1 / h\left|\partial_{j, h}^{2 r} u\right|_{0} \leqq C \ln 1 / h|u|_{2 r-2}
$$

Combining (7.16), (7.15) and (7.13) with (7.4) through (7.11) we obtain the desired result (7.3). Let us now briefly consider the inequality (7.2), août 1976. 
where $N=2$ and $r$ an odd integer, $r \geqq 3$. We proceed in the same manner as in the proof of (7.3) except that instead of (7.8) we can write

$$
\begin{aligned}
|\eta|^{-2} a_{1}^{\prime} j a_{0}= & {\left[\sum_{k=0}^{r-1}(-1)^{k}\left(\sin \eta_{2} / 2\right)^{2(r-1-k)}\left(\sin \eta_{2} / 2\right)^{2 k}\right] S^{2} /|\eta|^{2} } \\
& +|\eta|^{-2}\left(\sum_{l=1}^{2}\left(\sin \eta_{j} / 2\right)^{2 r}\right) \bar{g}_{j}(\eta)
\end{aligned}
$$

where $\vec{g}_{j}(\eta) \in C^{\infty}, \lim g_{j}(\eta)=0$ as $|\eta| \rightarrow 0$ and $S^{2}$ is defined Section 4. Hence instead of $(7.10)$ we obtain

$$
\begin{aligned}
& \int_{R^{N}} \hat{u}_{h} w\left(m_{0}-m_{1}\right) e^{i\langle\alpha, \eta\rangle} d \eta \\
& =(-1)^{r-1} h^{2 r-2} \sum_{k=0}^{r-2}(-1)^{k} \\
& \times \int_{R^{N}}\left(\widehat{\partial_{1, h}^{2(r-1-k)} \partial_{2, h}^{2 k}} u_{h}\right)\left(\frac{S^{2}}{|\eta|^{2}} w m_{1}\right) e^{i\langle\alpha, \eta\rangle} d \eta \\
& +(-1)^{r-1} h^{2 r-2} \sum_{j=1}^{2} \\
& \times \int_{R^{N}}\left(\widehat{\left.\partial_{j, h}^{2 r-2} u\right)_{h}} \sin ^{2} \eta_{j} / 2 \bar{g}_{j}(\eta)|\eta|^{-2} w m_{1} e^{i\langle\alpha, \eta\rangle} d \eta\right. \\
& +I_{2}=J_{1}+J_{2}+I_{2} \text {, }
\end{aligned}
$$

where $I_{2}$ has already been estimated in (7.11).

Since $w m_{1}\left(S^{2} /|\eta|^{2}\right) \in \mathscr{M}_{\infty}$ we obtain

$$
\left|J_{1}\right| \leqq C h^{2 r-2}|u|_{2 r-2}
$$

It is easily seen that $w m_{1} \sin ^{2} \eta_{j} / 2 \bar{g}_{j}(\eta)|\eta|^{-2} \in \mathscr{M}_{\infty}$ and hence

$$
\left|J_{2}\right| \leqq C h^{2 r-2}|u|_{2 r-2}
$$

Combining (7.18), (7.19), (7.20) and (7.11) we have

$$
\left|\int \hat{u}_{h} w\left(m_{0}-m_{1}\right) e^{i\langle\alpha, \eta\rangle} d \eta\right| \leqq C h^{2 r-2}|u|_{2 r-2} .
$$

In view of (7.4), (7.5), (7.7) and (7.21) we obtain the desired result (7.2), where the case $N=1$ is straightforward and therefore will not be presented. 


\section{SUPERCONVERGENCE AT INTERIOR MESH POINTS USING LOCAL AVERAGES}

Suppose that in a domain $\Omega, u_{h}$ is computed as an approximation to the solution $u$ of a boundary value problem using a Ritz-Galerkin method. Suppose further that on some domain $\Omega_{1} \subset \subset \Omega, u_{h}$ is of the form

$$
u_{h}=\sum C_{\alpha} \psi\left(h^{-1} x-\alpha\right)
$$

In practice, the $C_{\alpha}^{,} \mathrm{s}$ are first computed and then for fixed $x$, say for example at a mesh point $x=h \gamma \in \Omega_{0} \subset \subset \Omega_{1}$, the approximate solution is computed by forming

$$
u_{h}(h \gamma)=\sum C_{\alpha} \psi(\gamma-\alpha)=\sum C_{\alpha} a_{\gamma-\alpha}
$$

where all but a finite number of the $a_{\eta}$ vanish. In general, the best one to expect is that the error locally in maximum-norm is of order $h^{r}$. We shall show that if we restrict our attention to mesh points $h \gamma \in \Omega_{0}$, then under rather general conditions one can find constants say $d_{\eta}$ which are easily a priori computable (they depend only on $\psi$ but are independent of $h$ and $u$ ), all but a finite number of which in general vanish and $\sum C_{\alpha} d_{\gamma-\alpha}$ is in general closer to $u(h \gamma)$ than is $u_{h}(h \gamma)$ for any $h \gamma \in \Omega_{0}$. In order to construct such constants we need some preliminaries.

LEMMA 8.1: Let $t \geqq 1$ and $r \geqq 1$ be arbitrary but fixed integers. There exist uniquely determined real constants $k_{j}, j=0, \ldots, t-1$, which satisfy the linear algebraic system of equations

$$
\sum_{j=0}^{t-1} k_{j} \int_{R_{1}} \psi_{1}(y)(y+j)^{2 m} d y=\left\{\begin{array}{lll}
1 & \text { if } & m=0 \\
0 & \text { if } & m=1, \ldots, t-1,
\end{array}\right\}
$$

where $\psi_{1}(y)$ is the one dimensional B-spline of order $r$ defined in Section 2.

Proof: Since $\psi_{1}(y)$ is an even function and $\int_{R^{1}} \psi_{1}(y) d y=1$, the functions

$$
g_{m}(z)=\int_{R^{1}} \psi_{1}(y)(y+z)^{2 m} d y
$$

are monic polynomials of order $m$ in the variables $z^{2}$ and hence are linearly independent. Since the matrix $\left\{g_{m j}\right\}$ corresponding to the linear system (8.1) is given by $g_{m j}=g_{m}(j), j=0, \ldots, t-1, m=0, \ldots, t-1$, it follows that $\left\{g_{m j}\right\}$ is nonsingular which completes the proof.

Set

$$
k_{0}^{\prime}=k_{0}, \quad k_{j}^{\prime}=k_{j} / 2 \quad \text { and } \quad k_{-j}=k_{j} \quad \text { for } j=1, \ldots, t-1 .
$$

août 1976. 
Then the $k_{j}^{\prime}$ satisfies the equations

$$
\sum_{j=-(t-1)}^{t-1} k_{j}^{\prime} \int_{R^{1}} \psi_{1}(y)(y+j)^{2 m} d y=\left\{\begin{array}{lll}
1 & \text { if } & m=0, \\
0 & \text { if } & m=1, \ldots, t-1 .
\end{array}\right\}
$$

For $x \in R^{N}$ define

$$
K_{h}^{2 t}(x)=\prod_{l=1}^{N}\left[h^{-1} \sum_{j=-(t-1)}^{t-1} k_{j}^{\prime} \psi_{1}\left(h^{-1} x_{l}-j\right)\right] .
$$

LEMMA 8.2: Let $\Omega_{x}$ be the cube with sides of length $(2 t+r) h$ centered at $x$ then

$$
\left|u(x)-\left(K_{h}^{2 t} * u\right)(x)\right| \leqq C h^{2 t}|u|_{C^{2 t}\left(\Omega_{x}\right)},
$$

where $C$ is independent of $h$ and $u$.

Proof: Without loss of generality we may assume $x=0$. Note first that it follows from (8.4) and (8.3) in the case $m=0$ that $\int_{R^{N}} K_{h}^{2 t}(x) d x=1$. Hence

$$
u(0)-\left(K_{h}^{2 t} * u\right)(0)=\int_{R^{N}} K_{h}^{2 t}(y)[u(y)-u(0)] d y .
$$

Using Taylor's theorem we obtain

$$
\begin{aligned}
& \left|u(0)-\left(K_{h}^{2 t} * u\right)(0)\right| \\
& \quad \leqq\left|\sum_{1 \leqq|\alpha| \leqq t \sim 1} \frac{1}{|\alpha| !} D^{\alpha} u(0) \int_{R^{N}} K_{h}^{2 t}(y) y^{\alpha} d y\right|+C h^{2 t}|u|_{C_{2 t}\left(\Omega_{x}\right)} .
\end{aligned}
$$

We shall now show that the first term on the right hand side vanishes. Since $K_{h}^{2 t}(y)$ is an 'even function with respect to each variable $x_{l}$ separately, it is easily seen that in order to prove the lemma is sufficient to show that

$h^{-1} \int_{R_{1}} \sum_{j=-(t-1)}^{t-1} k_{j}^{\prime} \psi_{1}\left(h^{-1} z-j\right) z^{2 m} d z=0, \quad m=1, \ldots, t-1$.

But

$$
h^{-1} \int_{R^{1}} \sum_{j=-(t-1)}^{t-1} k_{j}^{\prime} \psi_{1}\left(h^{-1} z-j\right) z^{2 m} d z=\sum_{j=-(t-1)}^{t-1} k_{j}^{\prime} \int_{R^{1}} \psi_{1}(y)(y+j)^{2 m} d y
$$

and (8.5) follows from (8.3) which completes the proof.

Set $k_{j}^{\prime}=0$ for $j, \geqq t$ and for any $\beta \in Z^{N}$, let

$$
k_{\beta}=\prod_{j=1}^{N} k_{\beta_{j}}^{\prime}
$$

Revue Française d'Automatique, Informatique et Recherche Opérationnelle 
We may then write

$$
K_{h}^{2 t}(x)=h^{-N} \sum_{\beta \in Z^{N}} k_{\beta} \psi\left(h^{-1} x-\beta\right) .
$$

If $\gamma \in Z^{N}$ and in a neighborhood of the point $h \gamma$ we are given $v(x)$ of the form

$$
v(x)=\sum v_{\alpha} \psi\left(h^{-1} x-\alpha\right)
$$

then

$$
\begin{aligned}
\left(K_{h}^{2 t} * v\right)(h \gamma) & =\sum_{\alpha \in Z^{N}} v_{\alpha}\left[h^{-N} \sum_{\beta \in Z^{N}} k_{\beta}\left(\psi\left(\gamma-h^{-1} x-\beta\right), \psi\left(h^{-1} x-\alpha\right)\right)\right] \\
& =\sum_{\alpha \in Z^{N}} v_{\alpha}\left[\sum_{\beta \in Z^{N}} k_{\beta}(\psi(\gamma-\alpha-\beta-y), \psi(y))\right] \\
& =\sum_{\alpha \in Z^{N}} v_{\alpha}\left(\sum_{\beta \in Z^{N}} k_{\beta}(\psi * \psi)(\gamma-\alpha-\beta)\right) \\
& =\sum_{\alpha \in Z^{N}} v_{\alpha} d_{\gamma-\alpha},
\end{aligned}
$$

where

$$
d_{\eta}=\prod_{l=1}^{N} d_{\eta_{l}}^{\prime}=\prod_{l=1}^{N} \sum_{j=-(t-1)}^{t-1} k_{j}^{\prime}\left(\psi_{1} * \psi_{1}\right)\left(\eta_{l}-j\right)
$$

and $\left(\psi_{1} * \psi_{1}\right)(x)$ is the $B$-spline basis function of order $2 r$.

The $d_{\eta}$ are the constants referred to in the beginning of this section. Let us list some of their properties:

1) The $d_{\eta}$ are formed by products of the $d_{\eta_{j}}^{\prime}$ and hence may be easily computed from the one dimensional case.

2) All but a finite number of the $d_{\eta}$ vanish.

3) The $d_{\eta}$ are independent of $h$. They depend only on $\psi$ and $t$.

We are now in a position to prove a superconvergence type result at mesh points for the $L_{2}$ projection.

Let $\Omega_{0} \subset \subset \Omega_{1} \subseteq \Omega \subseteq R^{N}$. Let

$$
P_{0, \Omega} u=\sum u_{\alpha}^{0} \psi\left(h^{-1} x-\alpha\right)
$$

be the $L_{2}$ projection of $u$ onto $S^{h}(\Omega)$.

THEOREM 7: Let $t \geqq 1$ be an arbitrary but fixed integer and $\Omega_{1} \subset \subset \Omega_{1} \subset \Omega$ be any domain (which may depend on h) such that

$$
\operatorname{dist}\left(\Omega_{0}, \Omega_{1}\right) \geqq \frac{1}{2} \sqrt{N}(2 t+r) h .
$$

août 1976. 
Then for $h$ sufficiently small

$$
\sup _{h \gamma \in \Omega_{0}}\left|u(h \gamma)-\sum_{\alpha} u_{\alpha}^{0} d_{\gamma-\alpha}\right| \leqq C h^{2 t}|u|_{2 t, \Omega_{1}} .
$$

Here the fixed constants $d_{\eta}\left[\right.$ given by (8.8)] and $C$ are independent of $u, h, \Omega_{0}$ and $\Omega_{1}$.

REMARK: (8.9) says that by taking an appropriate linear combination of the computed coefficients $u_{\alpha}^{0}$ as approximation to $u$ one can achieve arbitrary order of accuracy at the mesh points.

Remark: The error estimate depends only on the values of $u$ in a $(1 / 2) / \sqrt{N}(2 t+r) h$ neighborhood of $\Omega_{0}$. In particular if we take $\Omega_{1}=\Omega$, then (8.9) gives us an estimate valid at mesh points whose distance from $\partial \Omega$ is $(1 / 2) / \sqrt{N}(2 t+r) h$.

Proof: Using (8.7) and (8.8) we have

$$
\begin{aligned}
\sup _{h \gamma \in \Omega_{0}}\left|u(h \gamma)-\sum_{\alpha \in Z^{N}} u_{\alpha}^{0} d_{\eta-\alpha}\right| \leqq & \sup _{h \gamma \in \Omega_{0}}\left|u(h \gamma)-\left(K_{h}^{2 t} * u\right)(h \gamma)\right| \\
& +\sup _{h \gamma \in \Omega_{0}} \mid\left(K_{h}^{2 t} *\left[u-P_{0, \Omega} u\right](h \gamma) \mid .\right.
\end{aligned}
$$

Now by Lemma 8.2 we have for $h$ sufficiently small that

$$
\sup _{h \gamma \in \Omega_{0}}\left|u(h \gamma)-\left(K_{h}^{2 t} * u\right)(h \gamma)\right| \leqq C h^{2 t}|u|_{2 t, \Omega_{1}}
$$

We shall show that the second term on the right of (8.10) vanishes. In fact for $h$ sufficiently small

$$
\begin{aligned}
\left(K_{h}^{2 t} *\left[u-P_{0, \Omega} u\right]\right)(h \gamma) & =\left(u(y)-P_{0, \Omega}(y), \sum_{\beta \in Z^{N}} d_{\beta} \psi\left(\gamma-h^{-1} y-\beta\right)\right) \\
& =\sum_{\eta \in Z^{N}} d_{\eta-\gamma}\left(u(y)-P_{0, \Omega}(y), \psi\left(h^{-1} y-\eta\right)\right)=0,
\end{aligned}
$$

which completes the proof.

We shall now prove an interior superconvergence result for Ritz-Galerkin methods. We shall need the following well known result for the $L_{2}$ and $H_{1}$ projections on $R^{N}$. They are proved using the duality argument of Nitsche.

Lemma 8.3: Let $u \in H^{r}$ then

$$
\left.\begin{array}{l}
\left\|u-P_{0} u\right\|_{-r} \leqq C h^{2 r}\|u\|_{r}, \\
\left\|u-P_{1} u\right\|_{2-r} \leqq C h^{2 r-2}\|u\|_{r}
\end{array}\right\}
$$


Let $u$ and $P_{1, \Omega}$ satisfy (6.2) where for $\Omega_{1} \subset \subset \Omega . P_{1, \Omega} u$ is of the form

$$
P_{1, \Omega} u(x)=\sum_{\alpha \in Z^{N}} u_{\alpha}^{1} \psi\left(h^{-1} x-\alpha\right) \quad \text { for } \quad x \in \Omega_{1} \text {. }
$$

THEOREM 8: Let $\Omega_{0} \subset \subset \Omega_{1}, u \in C^{2 r-2}\left(\Omega_{1}\right), r \geqq 3$ and $p$ be an arbitrary but fixed non-negative integer. Let $d_{\beta}$ be chosen according to (8.8) with $t=r-1$. Then if $N=1$ or $N=2$ and $r$ is odd

$$
\sup _{\gamma h \in \Omega_{0}}\left|u(h \gamma)-\sum_{\alpha \in Z^{N}} u_{\alpha}^{1} d_{\gamma-\alpha}\right| \leqq C\left(h^{2 r-2}|u|_{2 r-2, \Omega_{1}}+\|e\|_{-p, \Omega_{1}}\right) .
$$

In all other cases

$$
\sup _{\gamma h \in \Omega_{0}}\left|u(h \gamma)-\sum_{\alpha \in Z^{N}} u_{\alpha}^{1} d_{\gamma-\alpha}\right| \leqq C\left[h^{2 r-2}(\ln 1 / h)|u|_{2 r-2, \Omega_{1}}+\|e\|_{-p, \Omega_{1}}\right],
$$

where $C$ is a constant which is independent of $h$ and $u$ but in general depends on $\Omega_{0}, \Omega_{1}$ and $p$.

REMARK: If $r+1<l<2 r-3$ is a given integer, $u \in C^{l}\left(\Omega_{1}\right)$, and the $d_{\eta}$ are chosen with $t=l / 2$ if $l$ is even or $t=(l+1) / 2$ if $l$ is odd, then the same method of proof will yield

$$
\sup _{h \gamma \in \Omega_{0}}\left|u(h \gamma)-\sum u_{\alpha}^{1} d_{\gamma-\alpha}\right| \leqq C\left(h^{l}|u|_{l, \Omega_{1}}+\|e\|_{-p, \Omega_{1}}\right) .
$$

Proof: Let $\Omega_{0} \subset \subset \Omega_{0}^{\prime} \subset \subset \Omega_{0}^{\prime \prime} \subset \subset \Omega_{1}$. Then

$$
\begin{aligned}
\sup _{h \gamma \in \Omega_{0}}\left|u(h \gamma)-\sum_{\alpha \in Z^{N}} u_{\alpha}^{1} d_{\gamma-\alpha}\right| \leqq & \sup _{h y \in \Omega_{0}}\left|u(h \gamma)-\left(K_{h}^{2 r-2} * P_{0} \tilde{u}\right)(h \gamma)\right| \\
& +\left|K_{h}^{2 r-2} *\left(P_{0} \tilde{u}-P_{1} \tilde{u}\right)\right|_{0} \\
& +\left|K_{h}^{2 r-2} *\left(P_{1} \tilde{u}-P_{1, \Omega} u\right)\right|_{0, \Omega_{0}} .
\end{aligned}
$$

In view of (8.9):

$$
\sup _{h \gamma \in \Omega_{0}}\left|u(h \gamma)-\left(K_{h}^{2 r-2} * P_{0} \tilde{u}\right)(h \gamma)\right| \leqq C h^{2 r-2}|u|_{2 r-2, \Omega_{1}} .
$$

We note that

$$
\left|K_{h}^{2 r-2} *\left(P_{0} \tilde{u}-P_{1} \tilde{u}\right)\right|_{0} \leqq C\left|P_{0} \tilde{u}-P_{1} \tilde{u}\right|_{0},
$$

where $C$ is independent of $h$ and $u$. Hence from Theorem 6

$$
\begin{aligned}
& \left|K_{h}^{r-2} *\left(P_{0} \tilde{u}-P_{1} \tilde{u}\right)\right|_{0} \\
& \leqq C\left\{\begin{array}{lc}
h^{2 r-2}|u|_{2 r-2, \Omega_{1}} & \text { if } \begin{array}{c}
N=1 \text { or } N=2 \\
\text { and } r \text { is odd. }
\end{array} \\
h^{2 r-2}(\ln 1 / h)|u|_{2 r-2, \Omega_{1}} & \text { otherwise. }
\end{array}\right\}
\end{aligned}
$$

août 1976. 
Now

$$
\left|K_{h}^{2 r-2} *\left(P_{1} \tilde{u}-P_{1, \Omega} u\right)\right|_{0, \Omega_{0}} \leqq C\left|P_{1} \tilde{u}-P_{1, \Omega} u\right|_{0, \Omega_{0}^{\prime}}
$$

Now using Lemmas 6.1 and 8.3 we have, choosing $p \geqq 2 r$,

$$
\begin{aligned}
& \left|K_{h}^{2 r-2} *\left(P_{1} \tilde{u}-P_{1, \Omega} u\right)\right|_{0, \Omega_{0}} \\
& \quad \leqq C\left\|P_{1} \tilde{u}-P_{1, \Omega} u\right\|_{-p, \Omega_{0}^{\prime \prime}} \\
& \quad \leqq C\left(\left\|u-P_{1} \tilde{u}\right\|-2 r, \Omega_{0}^{\prime \prime}+\left\|u-P_{1, \Omega} u\right\|_{-p, \Omega_{0}^{\prime \prime}}\right) \\
& \left.\quad \leqq C h^{2 r}\|u\|_{r, \Omega_{1}}+\left\|u-P_{1, \Omega} u\right\|_{-p, \Omega_{1}}\right)
\end{aligned}
$$

The inequalities $(8.15),(8.16),(8.17)$ and (8.18) imply the desired result.

\section{SUPERCONVERGENCE AT INTERIOR MESH POINTS}

In this section we shall investigate the error $u(h \gamma)-P_{0 . \Omega}(h \gamma)$ and $u(h \gamma)-P_{1, \Omega}(h \gamma)$ at interior mesh points. We shall show that if $r$ is odd then the order of convergence at interior mesh points is "essentially" (for a more precise statement see Theorems 9 and 10) one order higher than predicted by Theorems 4 and 5 respectively.

Lemma 9.1: Let $u \in C^{0} \cap L_{2}$. Then

$$
P_{0} u(h \gamma)-u(h \alpha)=\frac{1}{(2 \pi)^{N}} \int \hat{u}_{h}\left[\tilde{\psi} m_{0}-1\right] e^{i\langle\gamma, \eta\rangle} d \eta
$$

with

$$
\tilde{\psi}(\eta)=\sum_{\beta \in Z^{N}} \psi(\beta) e^{-i\langle\beta, \eta\rangle}
$$

and $m_{0}$ is defined by (3.2).

Proof: We have that

$$
P_{0} u(h \gamma)=\sum_{\beta \in Z^{N}} u_{\beta}^{0} \psi(\gamma-\beta)
$$

with $u_{\beta}^{0}$ given by (3.2). Hence

$$
P_{0} u(h \gamma)=\frac{1}{(2 \pi)^{N}} \int_{R^{N}} \hat{u}_{h} \tilde{\psi} m_{0} e^{i\langle\gamma, \eta\rangle} d \eta
$$

The lemma now follows on noting that

$$
u(h \gamma)=\frac{1}{(2 \pi)^{N}} \int_{R^{N}} \hat{u}_{h} e^{i\langle\gamma, \eta\rangle} d \eta
$$


THEOREM 9. 1: Let $r \geqq 1$ be odd and $u \in C^{r+1} \cap L_{2}$. There exists a constant $C$ independent of $u$ and $h$ such that

$$
\sup _{\gamma \in Z^{N}}\left|P_{0} u(h \gamma)-u(h \gamma)\right| \leqq C h^{r+1}|u|_{r+1}
$$

Proof: Let $w \in C_{0}^{\infty}$ be such that $w(\eta)=1$ for $|\eta| \leqq \pi / 2$ and $w(\eta)=0$ for $|\eta|>\pi$. Then using Lemma 9.1 we may write

$$
\begin{array}{rl}
P_{0} & u(h \gamma)-u(h \gamma) \\
= & \frac{1}{(2 \pi)^{N}} \int_{R^{N}} \hat{u}_{h} w\left[\tilde{\psi} m_{0}-1\right] e^{i\langle\gamma \cdot \eta\rangle} d \eta \\
& +\frac{h^{r+1}}{(2 \pi)^{N}} \int_{R^{N}}\left(\Delta^{(r+1) / 2} u_{h}\right)(1-w) \frac{\left[\tilde{\psi} m_{0}-1\right]}{|\eta|^{r+1}} e^{i\langle\gamma, \eta\rangle} d \eta .
\end{array}
$$

Now

$$
\tilde{\psi} m_{0}-1=\tilde{\psi} m_{0}\left[1-\frac{\hat{\psi}}{\tilde{\psi}}\right]+\hat{\psi} m_{0}-1 \text {. }
$$

It is easy to see from Lemma 3.1 that

$$
\hat{\psi} m_{0}-1=\sum_{j=1}^{N} g_{j}(\eta)
$$

where the $g_{j}$ are entire functions and $g_{j}(\eta)=0\left(\eta_{j}^{2 r}\right)$ as $\eta_{j} \rightarrow 0$. Using the Poisson summation formula we have

$$
\tilde{\psi}(\eta)=\sum_{\beta \in Z^{N}} \hat{\psi}(\eta+2 \pi \beta)
$$

Then if $r \geqq 2$ and $|\eta| \leqq \pi / 2$

$$
\underset{\tilde{\psi}}{\hat{\psi}}=\prod_{j=1}^{N} \frac{1}{1+\left(\eta_{j}\right)^{r} \sum_{l \neq 0}(-1)^{l}\left(\eta_{j}+2 \pi l\right)^{-r}} .
$$

Now the function $\sum_{l \neq 0}(-1)^{l}\left(\eta_{j}+2 \pi l\right)^{-r}$ is an odd function and hence $\sum_{l \neq 0}(-1)^{l}\left(\eta_{j}+2 \pi l\right)^{-r}=O\left(\eta_{j}\right)$ as $\eta_{j} \rightarrow 0$. It then follows that for $|\eta| \leqq \pi / 2$

$$
1-\frac{\hat{\psi}}{\tilde{\psi}}=\sum_{j=1} G_{j}(\eta)
$$

where the $G_{j}(\eta)$ are analytic for $|\eta| \leqq \pi$ and $G_{j}(\eta)=O\left(\eta_{j}^{r+1}\right)$ as $\eta_{j} \rightarrow 0$. août 1976. 
Therefore

$$
w\left[\tilde{\psi} m_{0}-1\right]=\sum_{j=1}^{N} \eta_{j}^{r+1}\left(\frac{w f_{j}(\eta)}{\eta_{j}^{r+1}}\right)
$$

where $w f_{j} / \eta_{j}^{r+1}$ is in $C_{0}^{\infty}\left(R^{N}\right)$ and hence is in $\mathscr{M}_{\infty}$. Now for the first term on the right hand side of $(9.3)$ we obtain

$$
\begin{aligned}
& \left|\int_{R^{N}} \hat{u}_{h} w\left[\tilde{\psi} m_{0}-1\right] e^{i\langle\alpha, \eta\rangle} d \eta\right| \\
& \quad=\left|h^{r+1} \sum_{j=1} \int_{R^{N}}\left(\widehat{\frac{\partial^{r+1} u}{\partial x_{j}}}\right)_{h} w f_{j} / \eta_{j}^{r+1} e^{i\langle\alpha, \eta\rangle} d \eta\right| \\
& \quad \leqq C h^{r+1} \sum_{j=1}^{N}\left|\frac{\partial^{r+1} u}{\partial x_{j}^{r+1}}\right|_{0} .
\end{aligned}
$$

For the second term on the right hand side of (9.3), we observe that since $m_{0} \in \mathscr{M}_{\infty}$ and since $\tilde{\psi}(1-w) /|\eta|^{r+1} \in C^{\infty}$, it follows from Lemma 2.3 that $\tilde{\psi} m_{0}(1-w) /|\eta|^{r+1} \in \mathscr{M}_{\infty}$. Finally it is easy to see that

$$
(1-w) /|\eta|^{r+1} \in \mathscr{M}_{\infty}
$$

Hence the second term is estimated $C h^{r+1}|u|_{r+1}$ and the theorem follows in the case that $r \geqq 3$.

The case $r=1$ can be treated by elementary means. In this case the projection is totally local and

$$
u(\gamma h)-P_{0} u(\gamma h)=h^{-N} \int_{\left|x^{j}-\gamma^{j}\right| \leqq h / 2}[u(x)-u(h \gamma)] d x
$$

The assertion now follows using Taylors theorem.

We shall now prove a local version of Theorem 8 and then an analogous result for Ritz-Galerkin methods.

Let $\Omega_{0} \subset \subset \Omega_{1} \subset \subset \Omega$ and $P_{0, \Omega}$ be as is Section 5 .

THEOREM 9: Let $r$ be odd and $u \in C^{r+1}\left(\Omega_{1}\right) \cap L_{2}(\Omega)$. Then for all $h$ sufficiently small

$$
\sup _{\substack{h \alpha \in \Omega_{0} \\ \alpha \in Z^{N}}}\left|u(h \alpha)-P_{0, \Omega} u(h \alpha)\right| \leqq C h^{r+1}\left(|u|_{r+1, \Omega_{1}}+\|u\|_{0, \Omega}\right),
$$

where $C$ is independent of $h$ and $u$.

Revue Française d'Automatique, Informatique et Recherche Opérationnelle 
Proof: Let $\tilde{u}$ be as in the proof of Theorem 4 . Then

$$
\begin{aligned}
& \sup _{\substack{h \alpha \in \Omega_{0} \\
\alpha \in Z^{N}}}\left|u(h \alpha)-P_{0, \Omega} u(h \alpha)\right| \leqq \sup _{\substack{h \alpha \in \Omega_{0} \\
\alpha \in Z^{N}}}\left|\tilde{u}(h \alpha)-P_{0} \tilde{u}(h \alpha)\right| \\
& +\sup _{\substack{h \alpha \in \Omega_{0} \\
\alpha \in Z^{N}}}\left|P_{0} \tilde{u}(h \alpha)-P_{0, \Omega} u(h \alpha)\right| .
\end{aligned}
$$

In view of Theorem 8 we have

$$
\sup _{\substack{h \alpha \in \Omega_{0} \\ \alpha \in Z^{N}}}\left|\tilde{u}(h \alpha)-P_{0} \tilde{u}(h \alpha)\right| \leqq C h^{r+1}|\tilde{u}|_{r+1} \leqq C h^{r+1}|u|_{r+1, \Omega_{1}} .
$$

Using Lemma 5.1 with $\gamma=r+1$, it follows that

$$
\begin{aligned}
\sup _{\substack{\alpha h \in \Omega_{0} \\
\alpha \in Z^{N}}}\left|P_{0} \tilde{u}(h \alpha)-P_{0, \Omega} u(h \alpha)\right| & \leqq C h^{r+1}\left(\left\|P_{0} \tilde{u}-P_{0, \Omega} u\right\|_{0, \Omega_{1}}\right) \\
& \leqq C h^{r+1}\|u\|_{0, \Omega} .
\end{aligned}
$$

The desired result now follows.

THEOREM 10: Let $r \geqq 3$ be odd and $u \in C^{r+1}\left(\Omega_{1}\right)$. Then for all $h$ sufficiently small

$$
\begin{aligned}
& \sup _{\substack{h \alpha \in \Omega_{0} \\
\alpha \in Z^{N}}}\left|u(h \alpha)-P_{1, \Omega} u(h \alpha)\right| \\
& \quad \leqq C\left\{\begin{array}{ll}
\left(h^{4}(\ln 1 / h)|u|_{4, \Omega_{1}}+\left\|u-P_{1, \Omega} u\right\|_{-p, \Omega_{1}}\right), & r=3, \quad N \geqq 3 \\
h^{r+1}|u|_{r+1, \Omega_{1}}+\left\|u-P_{1, \Omega} u\right\|_{-p, \Omega_{1}}, & \text { otherwise. }
\end{array}\right\}
\end{aligned}
$$

Here $p$ is an arbitrary but fixed positive integer and $C$ is a constant which is independent of $u$ and $h$.

Proof: Let $\tilde{u}$ be as in the proof of Theorem 9. Then

$$
\begin{aligned}
& \sup _{\substack{h \alpha \in \Omega_{0} \\
\alpha \in Z^{N}}}\left|u(h \alpha)-P_{1, \Omega} u(h \alpha)\right| \\
& \quad \leqq \sup _{\alpha \in Z^{N}}\left|\tilde{u}(h \alpha)-P_{0} \tilde{u}(h \alpha)\right|+\left|P_{0} \tilde{u}-P_{1} \tilde{u}\right|_{0}+\left|P_{1} \tilde{u}-P_{1, \Omega} u\right|_{0, \Omega_{0}} .
\end{aligned}
$$

The first term on the right has been estimated in Theorem 9:

$$
\sup _{\alpha \in Z^{N}}\left|\tilde{u}(h \alpha)-P_{0} \tilde{u}(h \alpha)\right| \leqq C h^{r+1}|\tilde{u}|_{r+1} \leqq C h^{r+1}|u|_{r+1, \Omega_{1}} .
$$

In view of Theorem 6

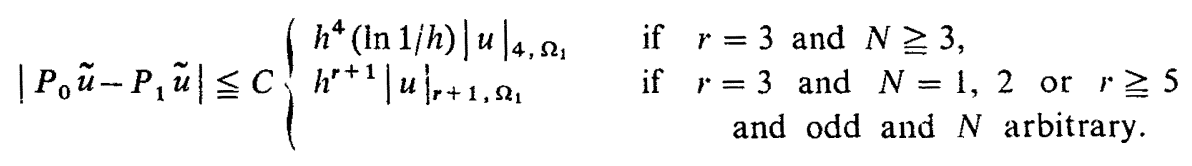

août 1976. 
Using Lemmas 6.1 and 8.3, it follows that for $\Omega_{0} \subset \subset \Omega_{0}^{\prime} \subset \subset \Omega_{1}$ :

$$
\begin{aligned}
\left|P_{1} \tilde{u}-P_{1, \Omega} u\right|_{0} & \leqq C\left\|P_{1} \tilde{u}-P_{1, \Omega} u\right\|_{-p, \Omega_{0}^{\prime}} \\
& \leqq C\left(\left\|\tilde{u}-P_{1} \tilde{u}\right\|_{2-r}+\left\|u-P_{1, \Omega}\right\|_{-p, \Omega_{0}^{\prime}}\right) \\
& \leqq C\left(h^{2 r-2}\|u\|_{r, \Omega_{1}}+\left\|u-P_{1, \Omega}\right\|_{-p, \Omega_{1}}\right) .
\end{aligned}
$$

Taken together, these inequalities imply (9.7), which completes the proof.

\section{THE NEUMANN PROBLEM}

Let $u$ satisfy

$$
\begin{gathered}
-\Delta u+u=f \quad \text { in } \Omega \\
\frac{\partial u}{\partial n}=0 \quad \text { on } \partial \Omega
\end{gathered}
$$

where $\Omega$ is a bounded domain with smooth boundary $\partial \Omega, \Delta$ is the Laplace operator and $\partial / \partial n$ is the outward normal derivative on $\partial \Omega$. The function $f$ is assumed to be in $H^{s}(\Omega)$ for appropriate $s$. It is well known that $u$ is characterized as the solution in $H^{1}(\Omega)$ of

$$
D(u, \varphi)+(u, \varphi)=(f, \varphi)
$$

for all $\varphi \in H^{1}(\Omega)$. Here $(u, \varphi)$ is the $L_{2}(\Omega)$ inner product and

$$
D(u, \varphi)=\sum_{j=1}^{N}\left(\frac{\partial u}{\partial x_{j}}, \frac{\partial \varphi}{\partial x_{j}}\right) .
$$

Now the Ritz-Galerkin approximation $u_{h} \in S^{h}(\Omega)$ to $u$ is characterized by

$$
D\left(u_{h}, \varphi\right)+\left(u_{h}, \varphi\right)=(f, \varphi)
$$

for all $\varphi \in S^{h}(\Omega)$. It was shown in [6] that the estimate

$$
\left\|u-u_{h}\right\|_{2-r, \Omega} \leqq C h^{r}\|f\|_{0, \Omega}
$$

is valid. Hence we may apply Theorem 5 (with $q=1$ and $P_{1, \Omega} u=u_{h}$ ) to obtain a maximum-norm estimate on an arbitrary compact subdomain $\Omega_{0}$ of $\Omega$. In Theorem 5 we take $p=2-r, r \geqq 3$ and combine (6.2) and (7.4) to obtain

$$
\left|u-u_{h}\right|_{0, \Omega_{0}} \leqq C h^{r}\left(|u|_{r, \Omega_{1}}+\|f\|_{0, \Omega}\right) \text {. }
$$


If we confine our attention to interior mesh points $h \gamma \in \Omega_{0}$, then combining (10.4) and (9.7) we obtain the following "superconvergence" result:

(i) If $r=3$ and $N=1,2$ or if $r \geqq 5$ is odd and $N$ arbitrary (but fixed)

$$
\sup _{h \gamma \in \Omega_{0}}\left|u(h \gamma)-u_{h}(h \gamma)\right| \leqq C h^{r+1}\left(|u|_{r+1, \Omega_{1}}+\|f\|_{0, \Omega}\right) .
$$

(ii) If $r=3$ and $N \geqq 3$ :

$$
\sup _{h \gamma \in \Omega_{0}}\left|u(h \gamma)-u_{h}(h \gamma)\right| \leqq C h^{4}(\ln 1 / h)\left(|u|_{4, \Omega_{1}}+\|f\|_{0}\right) .
$$

Now suppose that for $x \in \Omega, u_{h}$ is of the form $u_{h}=\sum C_{1}^{\alpha} \psi\left(h^{-1} x-\alpha\right)$. Let the constants $d_{\eta}$ be chosen satisfying (8.8) with $t=r-1$. Then from Theorem 8 we have the following:

(i) If $N=1$ and $r \geqq 3$ or $N=2$ and $r \geqq 3$ is odd

$$
\sup _{h \gamma \in \Omega_{0}}\left|u(h \gamma)-\sum C_{\alpha} d_{\gamma-\alpha}\right| \leqq C h^{2 r-2}\left(|u|_{2 r-2, \Omega_{1}}+\|f\|_{0}\right) \text {. }
$$

(ii) In all other cases when $r \geqq 3$ :

$$
\sup _{h \gamma \in \Omega_{0}}\left|u(h \gamma)-\sum C_{\alpha} d_{\gamma-\alpha}\right| \leqq C h^{2 r-2}(\ln 1 / h)\left(|u|_{2 r-2, \Omega_{1}}+\|f\|_{0}\right)
$$

\section{THE AUBIN-BABUŠKA PENALTY METHOD USING THE EXTRAPOLATION METHOD OF KING AND THE BABUŠKA LAGRANGE MULTIPLIER METHOD}

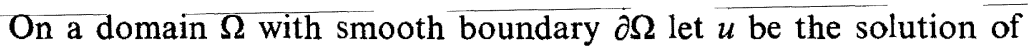

$$
\left.\begin{array}{rl}
-\Delta u+u=f & \text { in } \Omega, \\
u=0 & \text { on } \partial \Omega .
\end{array}\right\}
$$

In Aubin [1] and Babuška [4] a method was introduced for approximating the solution of (11.1) in which the trial function need not satisfy the boundary conditions and may be taken to be $S^{h}(\Omega)$. For this method the interior equations are the same as $(10.3)$ for the Neumann problem. The error estimates proved in [1] or [4] do not show that method is optimal in $H^{2-r}(\Omega)$; i. e., (10.4) was not proved. In King [11] an extrapolation method for the penalty method was given for which the estimate (10.4) remains valid. What is important to us here also is that the interior equations still remain to be (10.3) and the subspaces $S^{h}$ may be used. Hence in this case the estimate (10.5), (10.6), (10.7), (10.8) and (10.9) are valid.

In Babuška [3] another method was introduced for approximating solutions of (11.1). Here use is made of another set of approximating functions on $\partial \Omega$ 
which may be thought of as approximating $\partial u / \partial n$ ou $\partial \Omega$. These are independent of the trial functions on $\Omega$ which may be taken to be $S^{h}$. As before the important point for our purpose is that the interior equations are exactly the same as (10.3) and the estimate (10.4) holds. Hence the estimates (10.5), (10.6), (10.7), (10.8) and (10.9) are valid.

\section{ESTIMATES FOR THE GREEN'S FUNCTION}

For simplicity let us consider the Neumann problem of Section 10 and let $G(x, y)$ be the corresponding Green's function. Let $\bar{\Omega}_{0}$ and $\bar{\Omega}_{0}^{\prime}$ be two disjoint compact subdomains of $\Omega$. We shall consider $G(x, y)$ for $x \in \Omega_{0}$ and $y \in \Omega_{0}^{\prime}$; i. e. separated points in the interior of $\Omega$. The function $G$, for $y \in \Omega_{0}^{\prime}$ fixed satisfies

$$
D(G(., y), \varphi)+(G(., y), \varphi)=\varphi(y)
$$

for $\varphi \in H^{1}(\Omega) \cap C^{0}(\Omega)$. Now the Ritz-Galerkin Green's function corresponding to the approximation in Section 10 satisfies

$$
D\left(G_{h}(., y), \varphi\right)+\left(G_{h}(., y), \varphi\right)=\varphi(y)
$$

for $\varphi \in S^{h}(\Omega)$. Again we assume $r \geqq 3$. Now we want to estimate $G(x, y)-G_{h}(x, y)$ when $x \in \Omega_{0}$ and $y \in \Omega_{0}^{\prime}$, and $h$ is sufficiently small. For fixed $y \in \Omega_{0}^{\prime}$ we have

$$
D\left(G(., y)-G_{h}(., y), \varphi\right)+\left(G(., y)-G_{h}(., y), \varphi\right)=0
$$

for all $\varphi \in \dot{S}^{h}\left(\Omega_{1}\right)$, where $\Omega_{1}$ is such that $\Omega_{0} \subset \subset \Omega_{1}$ and $\overline{\Omega_{1}} \cap \overline{\Omega_{0}^{\prime}}$ is empty. Hence we may apply the estimate of Theorem 5 which yields

$$
\begin{aligned}
\left|G(x, y)-G_{h}(x, y)\right|_{0, \Omega_{0} \leqq} & C\left(h^{r}|G(., y)|_{r, \Omega_{1}}\right. \\
& \left.+\left\|G(., y)-G_{h}(., y)\right\|_{-p, \Omega_{1}}\right)
\end{aligned}
$$

for $p$ fixed but arbitrary. Clearly $\left.G(., y)\right|_{r, \Omega_{1}}$ is bounded.

In order to estimate the last term on right of (12.1) we observe that

$$
\begin{aligned}
\left\|G(., y)-G_{h}(., y)\right\|_{-p, \Omega_{1}} & \leqq i\left\|G(., y)-G_{h}(., y) \mid\right\|_{-p, \Omega} \\
& =\sup _{v \in C^{p}(\Omega)} \frac{\left|\left(G(., y)-G_{h}(., y), v\right)\right|}{\|v\|_{p, \Omega}} .
\end{aligned}
$$

Now for each $v \in C^{p}(\Omega)$ let $w$ be defined by

$$
\begin{aligned}
-\Delta w+w=v & \text { in } \Omega, \\
\frac{\partial w}{\partial n}=0 & \text { on } \partial \Omega .
\end{aligned}
$$


Then

$$
\left|\left(G(., y)-G_{h}(., y), v\right)\right|=\left|w(y)-w_{h}(y)\right|,
$$

where $\dot{w}_{h}$ is just the Ritz-Galerkin approximation to $w$ in $S^{h}(\Omega)$. Furthermore the estimate

$$
\|w\|_{p+2, \Omega} \leqq C\|v\|_{p, \Omega}
$$

is valid. Hence we obtain from (12.2), (12.3) and (12.4) that

$$
\left\|G(., y)-G_{h}(., y)\right\|_{-p, \Omega_{0}} \leqq C \sup _{w \in H^{p+2}(\Omega)} \frac{\left|w(y)-w_{h}(y)\right|}{\|w\|_{p+2, \Omega}} .
$$

Now since $y \in \Omega_{0}^{\prime}$ we may apply the interior estimate (12.5) to $w-w_{h}$; i. e.:

$$
\left|w-w_{h}\right|_{0, \Omega_{0}^{\prime}} \leqq C h^{r}\left(|w|_{r, \Omega_{0}^{\prime}}+\|v\|_{0, \Omega}\right)
$$

where $\Omega_{0}^{\prime} \subset \subset \Omega_{1}^{\prime}$ and $\bar{\Omega}_{1}^{\prime} \cap \bar{\Omega}_{0}^{\prime}$ is empty.

Clearly then for $p=r-2+[N / 2]+1$ we obtain by means of Sobolev's inequality

$$
\left|w-w_{h}\right|_{0, \Omega_{0}^{\prime}} \leqq C h^{r}\|w\|_{p+2, \Omega}
$$

Combining (12.5) and (12.6) we see that

$$
\left\|G(., y)-G_{h}(., y)\right\|_{-p, \Omega_{0}^{\prime}} \leqq C h^{r} .
$$

This together with $(12.6)$ yields

$$
\left|G(x, y)-G_{h}(x, y)\right| \leqq C h^{r}
$$

for $x \in \Omega_{0}$ and $y \in \Omega_{0}^{\prime}$.

\section{BOUNDARY VALUE PROBLEMS WITH NON-SMOOTH DATA}

As an application of the previous estimates we shall consider problems (10.1) and (10.2) in the case that the restriction of $f$ to $\Omega_{0}$ is assumed to belong to $L_{1}\left(\Omega_{0}\right)$ and outside of $\Omega_{0}, f$ is smooth. Without loss of generality in the following estimates we may assume that $f(x)=0$ for $x \in \Omega \backslash \Omega_{0}$. The solution $u$ to this problem is given by

$$
u(x)=\int_{\Omega} G(x, y) f(y) d y, \text { a. e. }
$$

août 1976. 
where $G$ is the Green's function introduced in Section 12. Now even though $f$ is not necessarily in $L_{2}(\Omega)$ the equations (10.3) make sense and there exists a unique solution $u_{h} \in S^{h}(\Omega)$ to this problem. Furthermore it is given by

$$
u_{h}(x)=\int_{\Omega} G_{h}(x, y) f(y) d y .
$$

Now let $\Omega_{0}^{\prime}$ be as in the previous section; i. e., $\Omega_{0}^{\prime} \subset \subset \Omega$ and $\Omega_{0} \cap \overline{\Omega_{0}^{\prime}}$ is empty. Then we have

$$
u(x)-u_{h}(x)=\int_{\Omega_{0}}\left(G(x, y)-G_{h}(x, y)\right) f(y) d y
$$

for $x \in \Omega_{0}^{\prime}$. By the estimate (12.7) we obtain immediately

$$
\left|u-u_{h}\right|_{0, \Omega_{0}^{\prime}} \leqq C h^{r} \int_{\Omega_{0}}|f(y)| d y .
$$

This shows that away from the singularities of $f$ the convergence rate in the interior of $\Omega$ is still as high as that of the smooth case. The analysis of Section 12 and 13 is not restricted to the Neumann problem. Exactly the same results hold for the two methods discussed in Section 11 applied to Dirichlet's problem and many other situations. The two important points in our applications are that the interior equations (6.1) be satisfied and that good estimates for negative norms of the error in a given approximation be available.

\section{REFERENCES}

1. J.-P. AuBIN, Approximation des problèmes aux limites non homogènes et régularité de la convergence, Calcolo, Vol. 6, 1969, pp. 117-139.

2. I. BABUŠKA, Approximation by Hill Functions, Comment. Math., Univ. Carolinae, Vol. 11, 1970, pp. 787-811.

3. I. BABUŠKA, The Finite Element Method with Lagranian Multipliers, Numer. Math., vol. 20, 1973, pp. 179-192.

4. I. BabuSKa, The Finite Element Method with Penalty, Math. Comp., Vol. 27, 1973, pp. 221-228.

5. J. H. Bramble and J. A. Nitsche and A. H. Schatz, Maximum Norm Interior Estimates for Ritz Galerkin Methods, Math. Comp., vol. 29, 1976.

6. J. H. BRAmble and J. E. Osborn, Rate of Convergence Estimates for NonSelfadjoint Eigenvalue Approximations, Math. Comp., Vol. 27, 1973, pp. 525-549.

7. P. L. ButZer and H. Berens, Semi-Groups of Operators and Approximation, Die Grundlehren der math. Wissenschaften, Band 145, Springer-Verlag, New York, 1967.

8. C. DE BOOR and G. FIX, Spline Approximation by Quasi-Interpolants, J. Approximation Theory, vol. 8, 1973, pp. 19-45. 
9. F. D. Guglielmo, Construction d'approximations des espaces de Sobolev sur des réseaux en simplexes, Calcolo, Vol. 6, 1969, pp. 279-331.

10. G. Fix and G. Strang, A Fourier Analysis of the Finite Element Method, Proc. CIME Conference, 1971, Cremonese, Rome (to appear).

11. J. T. KING, New Error Bounds for the Penalty Method and Extrapolation, Numer. Math., vol. 23, 1974, pp. 153-165.

12. J. A. Nirsche and A. H. Schatz, On Local Approximation Properties of $L_{2}$ Projection on Spline Subspaces, Applicable Analysis, Vol. 2, No. 2, July 1972.

13. J. A. Nitsche, Interior Estimates for Ritz Galerkin Methods (preprint).

14. I. J. Schoenberg, Approximation with Special Emphasis on Spline Functions, Academic Press, New York, London, 1969.

15. E. M. Stein, Singular Integrals and Differentiability Properties of Functions, Princeton University Press, Princeton, New Jersey, 1970.

16. A. Zygmund, Trigonometrical Series, Vol. 2, Cambridge, England, 1959. 